School of Social Sciences

Economics Division

University of Southampton Southampton SO17 1BJ , UK

Discussion Papers in

Economics and Econometrics

Fairness and Desert in Tournaments

David Gill \& Rebecca Stone

No. 0903

This paper is available on our website

http://www.southampton.ac.uk/socsci/economics/research/papers 


\title{
Fairness and Desert in Tournaments
}

\author{
David Gill * \\ Rebecca Stone \\ Division of Economics \\ School of Law \\ University of Southampton \\ New York University
}

This Version: 6 October 2009

\begin{abstract}
We model the behavior of agents who care about receiving what they feel they deserve in a two-player rank-order tournament. Perceived entitlements are sensitive to how hard an agent has worked relative to her rival, and agents are loss averse around their meritocratically determined endogenous reference points. In a fair tournament sufficiently large desert concerns drive identical agents to push their effort levels apart in order to end up closer to their reference points on average. In an unfair tournament, where one agent is advantaged, the equilibrium is symmetric in the absence of desert, but asymmetric in the presence of desert. We find that desert concerns can undermine the standard conclusion that competition for a fixed supply of status is socially wasteful and explain why, when the distribution of output noise is fat-tailed, an employer might use a rank-order incentive scheme.
\end{abstract}

Keywords: Desert, Equity, Tournament, Loss Aversion, Reference-Dependent Preferences, Reference Point, Psychological Game Theory, Status, Relative Performance Evaluation

JEL Codes: D63, J33

* Corresponding author: David Gill, Division of Economics, School of Social Sciences, University of Southampton, Southampton SO17 1BJ, United Kingdom. Email: d.gill@soton.ac.uk. Phone: +44 (0) 7714756170 . 


\section{Introduction}

Rank-order tournaments, where agents compete for a fixed prize or set of prizes, are ubiquitous. Promotional tournaments are common in consulting, law partnerships, academia and industry. Firms frequently use bonus schemes based on relative performance. Sports contests, examinations, patent races, elections and competition for status can all be thought of as tournaments. We claim that in a competitive setting such as a tournament, agents often care about receiving their 'just deserts'. We adopt a meritocratic notion of desert according to which an agent's perceived entitlement will be sensitive to how hard she has worked relative to her rival. Desertmotivated agents will feel hard done by if they receive less than what they perceive to be their fair recompense, while feelings of elation or guilt are possible if they do better than they deserve. We analyze how the introduction of desert alters equilibrium play and payoffs in tournaments, and we apply our findings to analyze competition for status/rank and the design of incentive schemes.

While little work has been carried out to formally introduce desert concerns into a theoretical economic model, ${ }^{1}$ there exists an empirical literature which supports the idea that people are indeed motivated by a meritocratic notion of desert. According to a review of relevant literature by Konow (2003), "a common view is that differences owing to birth, luck and choice are all unfair and that only differences attributable to effort are fair" (pp. 1207-1208). Furthermore, Konow (1996) distills an accountability principle from the responses to his attitude survey according to which a person's entitlement varies in direct proportion to the value of his relevant discretionary variables, relative to others (p.19). This is closely related to the claims of equity theory, a social psychological theory of fairness that has its origins in Aristotle's claim that the equitable ratio of outcomes is proportional to the ratio of inputs (Konow, 2003). In its modern manifestation equity theory goes back to Adams (1965), whose work led him to conclude that "when [a person] finds that his outcomes and inputs are not in balance in relation to those of others, feelings of inequity result" (p. 280) and that "there can be little doubt that inequity results in dissatisfaction, in an unpleasant emotional state, be it anger or guilt" (p. 283). The significance of equity theory is also noted by Rabin (1998) who writes that "desert will obviously be relevant in many situations - and the massive psychological literature on 'equity theory' shows that people feel that those who have put more effort into creating resources have more claim on those resources" (p. 18).

Experimental economics provides further evidence in favor of the idea that people are sensitive to considerations of desert. In a bargaining experiment, Burrows and Loomes (1994) find

1 An exception is Konow (2000) who develops a model in which people are influenced by the departure of the allocation from a fair one which reflects the efforts exerted by all agents. However, Konow considers only the optimal division of output by a dictator given efforts, and not the choice of effort levels by the agents. 
that bargained outcomes tend to exhibit greater inequality, awarding higher final payoffs to the party that began with a greater initial endowment, when endowments were allocated according to parties' performances in a simple word game than when the endowments were allocated at random. They conclude that the results of their experiment are consistent with the proposition that "many people believe that when different individuals have a similar ability and opportunity to put in effort, those that put in more effort should get a greater reward because they are relatively deserving" (p. 220). Konow (2000), Frohlich et al. (2004) and Cappelen et al. (2007) provide evidence that, in their allocation decisions, dictators are sensitive to agents' relative efforts or investments. Frohlich et al. state that "the just deserts response is modal" (p. 109). ${ }^{2}$

We suppose that two agents compete in a Lazear and Rosen (1981) type simultaneous-move rank-order tournament and that each agent is sensitive to deviations of her monetary payoff from an endogenous reference point given by her perceived entitlement. We assume that these desert payoffs are more steeply increasing in the loss domain than the gain domain. This captures the central stylized fact - loss aversion - that has emerged from the empirical literature on referencedependent preferences more generally (Kahneman and Tversky, 1979; see also Rabin, 1998, for a survey). ${ }^{3}$ As one agent's undeserved bonus has meant another's undeserved loss, we might expect any sensation of elation the agent experiences as a result of having her reference point exceeded to be somewhat muted. Indeed, our model permits desert guilt, whereby exceeding the reference point is painful.

We consider fair tournaments, in which the agents are identical, and unfair tournaments, in which one agent is given an advantage. In a fair tournament, or an unfair one in which the advantage is felt to be fully deserved, the reference point is given by the agent's expected payoff given the chosen effort levels. ${ }^{4}$ The idea is that an agent's average payoff is a reflection of the useful effort she has exerted relative to her rival and thus plausibly represents the proportion of the prize that she feels she deserves. In an unfair tournament in which the advantage is not felt to be fully deserved, the reference point is given by the counterfactual expected payoff had the advantage been as big as is felt to be deserved.

If desert payoffs are falling in the gain domain, then desert preferences will be structurally similar to the preferences of the inequity-averse agents of Fehr and Schmidt (1999). ${ }^{5}$ However, desert payoffs are defined with respect to a different reference point. Inequity averse agents

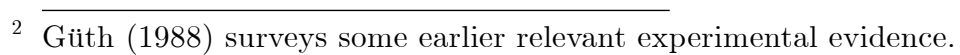

${ }^{3}$ In the specific context of fairness judgments, Kahneman et al. (1986) find strong evidence for loss aversion. People apparently perceive that it is more important that firms avoid hurting customers relative to a "fair" reference transaction than that they attempt to increase customer surplus relative to this reference level.

4 Experimental evidence from the psychology literature supports the thesis that agents' emotional responses when receiving the payoff associated with a lottery depend on expectations. See, for instance, Mellers (1999).

5 Bolton and Ockenfels (2000) develop a similar theory of inequity aversion. 
exhibit a preference for equality of payoffs across agents in a reference group: when there are only two agents in the group, each agent's reference point is simply the payoff of the other agent. By contrast, in our setup reference points are functions of agents' effort levels. In this sense, our agents adopt a more sophisticated conception of fairness than those of Fehr and Schmidt. They care about the relationship between the distribution of material payoffs and the distribution of agents' efforts, not just about the brute distribution of material payoffs. ${ }^{6}$

The structure of our agents' preferences also clearly resembles that of other models of reference-dependent preferences such as Kahneman and Tversky (1979) and Köszegi and Rabin (2006). However, the reference point of the value function in Kahneman and Tversky's (1979) Prospect Theory is exogenous, while our reference points are endogenously determined (since they depend on agents' efforts). Köszegi and Rabin's (2006) agents are sensitive to deviations from their endogenous expectations, but they consider only a single agent problem and the reference point is not sensitive to agents' actual choices, instead depending on prior expectations. Models of disappointment aversion (e.g., Bell, 1985; Loomes and Sugden, 1986; Kőszegi and Rabin, 2007, Sect. IV) allow the expectations-based reference point to depend on an agent's chosen action. Assuming elation rather than desert guilt, and when the reference point is given by the expected payoff-which will be the case in fair tournaments or unfair tournaments where each agent's (dis)advantage is felt to be deserved - our model can be interpreted as an extension of disappointment aversion to strategic situations in which agents compete. Gill and Prowse (2009) provide experimental evidence for disappointment aversion in a sequential-move tournament. However, in unfair tournaments in which each agent's relative (dis)advantage is not felt be to be deserved, desert and disappointment aversion come apart, which suggests a way to distinguish desert and disappointment aversion experimentally, a possibility discussed in Section 4.2 .

\section{$1.1 \quad$ Summary of Findings}

In the absence of desert, there is a unique and symmetric Nash equilibrium in both fair and unfair tournaments, as winning probabilities are a function of the difference in the agents' efforts in a Lazear and Rosen (1981) type tournament.

In a fair tournament, weak desert concerns do not affect this equilibrium. However, sufficiently large desert concerns drive the identical agents to push their efforts apart. Intuitively, when the agents exert the same effort, one ends up winning and the other losing even though

6 Extending Fehr and Schmidt's notion of inequity aversion to allow aversion to differences in payoffs net of effort costs, as done by Demougin and Fluet (2003) in the context of tournaments, implies that, unlike in our set-up, the payoff of the other agent enters into the reference point (together with the difference in effort costs). 
neither is more deserving than the other. The greater the difference in the agents' efforts, the greater the probability that an outcome emerges in which the more hardworking and therefore more deserving agent wins and the closer on average are both agents' reference points to their actual payoffs.

In an unfair tournament, the equilibrium can no longer be symmetric: the advantaged agent has a stronger marginal incentive to exert effort as she is more likely to win and so suffers less in expectation from increasing her deserved reference point. In a linear example, we show that as the strength of desert preferences, the size of the advantage and the extent to which the advantage is felt to be deserved go up, the difference between the advantaged and disadvantaged agents' efforts increases.

We develop two applications of our model. First, we find that desert concerns can undermine the standard conclusion that competition for a fixed supply of status is socially wasteful. Second, we turn to an employer's choice of relative performance compensation scheme. We find that an employer prefers a tournament to relative performance pay linear in the difference in employees' outputs when the distribution of output noise is sufficiently fat-tailed, thus providing a new explanation for why employers might choose to use tournament-style incentive schemes.

The paper proceeds as follows. Section 2 sets out the model. Section 3 derives general results. Section 4 presents a linear example. Section 5 considers the two applications. Section 6 concludes. Omitted proofs are provided in the appendix.

\section{Model}

Two agents are competing to win a fixed prize of monetary value $v>0$ in a Lazear and Rosen (1981) type rank-order tournament. ${ }^{7}$ (Later, in Section 5 , the prize will be chosen optimally by a firm.) The agents simultaneously choose effort levels $e_{i} \in[0, \infty)$ and share a twice continuously differentiable cost function $C\left(e_{i}\right)$, where $C(0)=0, C^{\prime}(0) \geq 0, C^{\prime}\left(e_{i}\right)>0$ for $e_{i}>0$, and $C^{\prime \prime}\left(e_{i}\right)>0$. Agent $i^{\prime} s$ output is given by $\psi_{i}=e_{i}+a_{i}+\epsilon_{i}$ where the noise term $\epsilon_{i} \backsim f\left(\epsilon_{i}\right)$ with $E\left[\epsilon_{i}\right]=0$ and $\operatorname{Var}\left[\epsilon_{i}\right]=\sigma^{2}$. The $\epsilon_{i}$ 's are i.i.d. across the two agents. The agent with the higher output wins the prize. Let $k \equiv a_{i}-a_{j} \geq 0$ represent the advantage enjoyed by agent $i$. In a fair tournament, $k=0$. In an unfair tournament $k>0 .{ }^{8}$

7 Introducing a prize for the loser has no effect on the analysis, as all payoffs are increased by a constant. $v$ would then be the difference between the winner's prize and the loser's prize.

8 Without loss of generality, agent $i$ is always the advantaged agent. We adopt the fair / unfair terminology as it is standard in the literature (an uneven tournament is one in which the agents have different costs of effort): we do not intend that these terms denote how the agents perceive the advantage. Tournaments might be unfair because of discrimination (e.g., affirmative action) or ability differences which do not affect marginal productivity (note we can think of $e_{i}=0$ as a minimum level of effort, which produces a different amount for different agents), or because the agents have already produced some output at an initial stage. 
Let $P_{i}\left(e_{i}, e_{j}\right)$ represent $i$ 's probability of winning and let $\eta \equiv \epsilon_{j}-\epsilon_{i}$. As the $\epsilon_{i}$ 's are i.i.d., $\eta \backsim q(\eta)$ with $E[\eta]=0, \operatorname{Var}[\eta]=2 \sigma^{2}$ and $q(\eta)$ is symmetric about zero. We assume that the c.d.f. of $\eta, Q(\cdot)$, is twice continuously differentiable, that $q(\eta)>0 \forall \eta$, and that $v q(k)>C^{\prime}(0)$. Then:

$$
\begin{aligned}
P_{i}\left(e_{i}, e_{j}\right) & =\operatorname{Pr}\left[\psi_{i} \geq \psi_{j}\right]=\operatorname{Pr}\left[e_{i}+a_{i}+\epsilon_{i} \geq e_{j}+a_{j}+\epsilon_{j}\right] \\
& =\operatorname{Pr}\left[e_{i}-e_{j}+k \geq \eta\right]=Q\left(e_{i}-e_{j}+k\right) \in(0,1) ; \\
P_{j}\left(e_{i}, e_{j}\right) & =1-P_{i}\left(e_{i}, e_{j}\right)=1-Q\left(e_{i}-e_{j}+k\right) .
\end{aligned}
$$

We capture the agents' desert concerns by supposing that each cares not only about her monetary payoff $y_{i}$, but also about the comparison of this payoff with an endogenous reference point $r_{i}$ that represents the payoff that she perceives that she deserves. Our notion of desert is meritocratic in the sense that the harder an agent works relative to her rival, the more she feels she deserves. Thus, $r_{i}\left(e_{i}, e_{j}\right)$ is a function of both agents' efforts, and increases in agent $i^{\prime} s$ own effort and decreases in her rival's effort:

$$
\frac{\partial r_{i}\left(e_{i}, e_{j}\right)}{\partial e_{i}}>0 ; \quad \frac{\partial r_{i}\left(e_{i}, e_{j}\right)}{\partial e_{j}}<0
$$

We assume that the agents share a common notion of desert, so the agents agree on what each deserves. Let $\theta \in[0,1]$ represent the degree to which any advantage for agent $i$ is felt to be deserved, so the deserved advantage is given by $\theta k$. In a fair tournament $(k=0)$, or an unfair tournament where agent $i^{\prime} s$ advantage is felt to be fully undeserved $(k>0, \theta=0)$, an agent feels she deserves more than her rival if and only if she works harder:

$$
r_{i} \gtreqless r_{j} \Leftrightarrow e_{i} \gtreqless e_{j} .
$$

In an unfair tournament where the advantage is felt to be partially or fully deserved $(k>0, \theta>0)$, the advantaged agent $i$ feels she deserves more when she works as hard as her rival, while the disadvantaged agent feels she deserves less, i.e., $e_{i}=e_{j} \Rightarrow r_{i}>r_{j}$. Furthermore, when $e_{i}=e_{j}$, $r_{i}$ is increasing in $\theta$ while $r_{j}$ falls in $\theta$.

To operationalize these notions, we assume that the agents feel they deserve their expected monetary payoff conditional on the chosen efforts and any advantage being as big as is felt to be deserved. Let

$$
\widetilde{P}_{i}\left(e_{i}, e_{j}\right) \equiv Q\left(e_{i}-e_{j}+\theta k\right)
$$


be agent $i^{\prime} s$ probability of winning under the deserved advantage, so:

$$
\begin{aligned}
r_{i}\left(e_{i}, e_{j}\right) & =v \widetilde{P}_{i}\left(e_{i}, e_{j}\right) \\
r_{j}\left(e_{i}, e_{j}\right) & =v \widetilde{P}_{j}\left(e_{i}, e_{j}\right)=v\left(1-\widetilde{P}_{i}\left(e_{i}, e_{j}\right)\right) .
\end{aligned}
$$

In a fair tournament $(k=0)$ or an unfair one where the advantage is felt to be fully deserved $(k>0$ and $\theta=1), P_{i}=\widetilde{P}_{i}$ so the reference point is given by the actual expected payoff given the chosen efforts, i.e., $r_{i}=E\left[y_{i}\right]=v P_{i}$. In a fair tournament, $e_{i}=e_{j} \Rightarrow r_{i}=r_{j}=\frac{v}{2}$. When the tournament is unfair and the advantage is not felt to be fully deserved $(k>0, \theta<1)$, the reference point is based on a counterfactual thought experiment. ${ }^{9}$

The agents are assumed to be loss averse around their endogenous reference points. In particular, each agent's instantaneous utility is assumed to be separable in money, desert and effort cost as follows:

$$
U_{i}\left(y_{i}, e_{i}, e_{j}\right)=y_{i}+D_{i}\left(y_{i}-r_{i}\left(e_{i}, e_{j}\right)\right)-C\left(e_{i}\right)
$$

where the desert payoff $D_{i}(x)$ has the following piecewise linear reference-dependent form: $D_{i}(x)=g x$ if $x>0 ; D_{i}(x)=0$ if $x=0$; and $D_{i}(x)=l x$ if $x<0$.

$l x$ represents the payoff associated with situations in which $y_{i}<r_{i}$ and the agent receives less than she deserves. In that case we say that the agent suffers a desert loss and we assume that such losses are unambiguously painful, so $l>0$.

$g x$ represents the payoff associated with situations in which $y_{i}>r_{i}$ and the agent receives more than she deserves. $g$ can be positive or negative depending on whether the agent's preferences exhibit desert elation or desert guilt. (We suggest that elation is likely to be more prevalent when agents positively choose to enter the tournament, e.g., professional poker players, or when the interaction is anonymous). We restrict $g>-1$ to avoid giving the tournament winner an incentive to forgo part of her monetary payoff to reduce guilt (either by burning money or making a transfer to the loser).

Let $\lambda \equiv l-g$. The assumption of loss aversion implies that $\lambda>0$, i.e., $l>g$, so desert losses resonate more strongly than any desert elation, as is consistent with Prospect Theory

9 This expectations-based framework is tractable, incorporates advantages in a natural way and allows changes in the reference point to reflect the productivity of effort to the agent (in terms of its marginal impact on winning probabilities). When the tournament is fair, or the tournament is unfair but each agent's (dis)advantage is felt to be fully deserved, our model can also be interpreted as an extension of disappointment aversion to situations where agents compete. A disadvantage is that $r_{i}$ bears no relationship to the cost of effort, but, given we restrict attention to common cost functions and the agents choose how hard to work, it is plausible that productivity of effort should constitute the main criterion for determining desert. We also note that the analysis would be unaltered if the agents compared monetary payoffs net of effort costs to the relevant expectation net of effort costs, as the effort costs would cancel in the comparison. 
(see Kahneman and Tversky, 1979, p. 279). The value of $\lambda$ measures the strength of desert. The above entails the following formulation for expected utility:

$$
\begin{aligned}
E U_{i}\left(e_{i}, e_{j}\right) & =P_{i}\left[v+g\left(v-v \widetilde{P}_{i}\right)\right]+\left(1-P_{i}\right)\left[0+l\left(0-v \widetilde{P}_{i}\right)\right]-C\left(e_{i}\right) \\
& =v P_{i}-v \lambda P_{i}\left(1-\widetilde{P}_{i}\right)-v l\left(\widetilde{P}_{i}-P_{i}\right)-C\left(e_{i}\right) .
\end{aligned}
$$

We restrict attention to pure strategy Nash equilibria, which we call desert equilibria. Taking her rival's effort as given, each agent chooses her own effort to maximize her expected utility $E U_{i}\left(e_{i}, e_{j}\right)$. Having exerted their chosen effort levels, the agents receive their monetary payoffs and also observe the effort level exerted by the other agent. ${ }^{10}$ Implicitly, we are assuming that the problem of moral hazard precludes the agents' use of insurance and that narrow framing of losses and gains (see Barberis et al., 2006) prevents the agents from diversifying the variability of their desert payoffs across multiple tournaments or other events.

\subsection{Relationship to Psychological Game Theory}

Although we have modelled desert using standard game theory, our game is psychological (Geanakoplos et al., 1989) as agent $i^{\prime} s$ utility depends on her belief about the effort of agent $j$ via the reference point. In particular, our game falls under Battigalli and Dufwenberg's (2009) framework of a dynamic psychological game as utility depends on terminal node (ex post) beliefs, so beliefs can update during the course of the game (in contrast to Geanakoplos et al., in which utilities only depend on initial beliefs). As we have assumed that the agents observe each other's efforts ex post, the actual efforts pin down these beliefs at the terminal nodes. This explains why we do not need to introduce the apparatus of psychological games: we can write payoffs as a function of actions alone, given the actions determine the first-order beliefs.

Suppose, on the other hand, that the agents could not observe each other's efforts. Then payoffs would depend on terminal node beliefs which in turn would depend on the agents' inferences. However, restricting attention to pure strategy Nash equilibria of the one-shot game, as we do throughout the paper, the equilibria are the same as with observation. To see this, note that with or without observation the agents' optimization problem is equivalent: in equilibrium, agent $i$ optimizes given $e_{j}$ and a correct terminal node belief arising from this $e_{j}$. Whether this

\footnotetext{
${ }^{10}$ Although we focus on the case where efforts are ex post observable, we note in Section 2.1 that in the absence of observation the set of pure strategy Nash equilibria of our one-shot game would remain the same. In any case, observation is a reasonable assumption in a number of settings. In some work environments, workers can collect credible (but perhaps, from the employer's perspective, legally unverifiable) information from other co-workers or direct observation about how hard their rival has worked. In a sports contest, e.g., a golf tournament, televised replays or spectator reports might give observability. At the end of a patent race or election, the rival's spending often becomes public.
} 
belief comes from a direct observation or an inference which in equilibrium must be correct is technically immaterial (the noise draws have full support, so any tournament outcome and set of outputs are consistent with a given equilibrium).

If we wanted to consider mixed strategies or repeated play in the absence of ex post observation, Battigalli and Dufwenberg's (2009) framework would have to be introduced explicitly. With mixing, each agent would form an ex post distribution of beliefs over their rival's effort as a function both of the ex ante mixing distribution and the outcome of the tournament, and would anticipate this when choosing how hard to work. With repeated play, the absence of observation would affect responses to a rival's deviation as such deviations would not be observable directly.

Other psychologically motivated preferences have been modelled using psychological game theory. For example, higher-order beliefs are fundamental to modelling reciprocity (Rabin, 1993; Dufwenberg and Kirchsteiger, 2004; Falk and Fischbacher, 2006) and guilt aversion (Battigalli and Dufwenberg, 2007). Guilt aversion depends on beliefs about how much you let others down relative to what they initially expected (simple guilt) or about the terminal node beliefs of others about the extent to which you let them down (guilt from blame). In contrast, our notion of desert guilt from doing better than deserved depends only on first-order beliefs. Köszegi and Rabin's (2006) model of reference-dependent preferences is also technically a psychological game, but one in which utilities depend only on initial beliefs, rather than terminal node beliefs as in our model of desert.

\section{General Results}

\subsection{No Desert}

We start by showing that in the absence of desert, all (pure strategy) Nash equilibria must be symmetric and unique. The result is known in the literature, but we repeat it here to provide a clear comparator for our later results. To understand the result, note that at any $\left(e_{i}, e_{j}\right)$ pair the agents face the same marginal impact of effort on their probability of winning:

$$
\begin{aligned}
& \frac{\partial P_{i}\left(e_{i}, e_{j}\right)}{\partial e_{i}}=\frac{\partial Q\left(e_{i}-e_{j}+k\right)}{\partial\left(e_{i}-e_{j}\right)}=q\left(e_{i}-e_{j}+k\right) ; \\
& \frac{\partial P_{j}\left(e_{i}, e_{j}\right)}{\partial e_{j}}=\frac{-\partial Q\left(e_{i}-e_{j}+k\right)}{\partial\left(e_{i}-e_{j}\right)}(-1)=q\left(e_{i}-e_{j}+k\right) .
\end{aligned}
$$

This is because an increase in $e_{i}$ is equivalent to a decrease in $e_{j}$ as winning probabilities depend on $e_{i}-e_{j}$, while the impact of $e_{i}$ on $P_{i}$ is the opposite of its impact on $P_{j}$ as $P_{i}=1-P_{j}$. The first order conditions then give any symmetric equilibrium as $e_{i}^{*}=e_{j}^{*}=C^{\prime-1}(v q(k))$. The assumption that $v q(k)>C^{\prime}(0)$ ensures that if one agent exerts zero effort, the other has a strict 
incentive to work, so all symmetric equilibrium efforts are interior. Asymmetric equilibria are not possible, as at any $\left(e_{i}, e_{j}\right)$ pair the agents' marginal impact of effort on winning probabilities $q\left(e_{i}-e_{j}+k\right)$ are identical, as explained above. ${ }^{11}$

Proposition 1 In the absence of desert $(l=g=\lambda=0)$, any pure strategy Nash equilibrium will be unique, symmetric and interior, and will be given by $e_{i}^{*}=e_{j}^{*}=C^{\prime-1}(v q(k))$.

As noted by Lazear and Rosen (1981) and by Nalebuff and Stiglitz (1983), for the case with $k=0$ even if the local second-order conditions are satisfied, a pure strategy equilibrium may not exist (if $\sigma^{2}$ is too low, $v q(0)$ may be so high that at the local symmetric equilibrium the agents prefer to deviate to zero effort). When $q$ is unimodal (e.g., $\eta$ is normally distributed), equilibrium efforts fall in the size of the advantage $k$ : the advantage demotivates both agents equally.

In the absence of desert, introducing risk aversion over monetary payoffs (as done by Nalebuff and Stiglitz, 1983) does not change qualitative behavior, so our simple tournament structure nicely disentangles the effects of desert from those of risk aversion. Assuming that $U_{i}=\phi\left(y_{i}\right)-$ $C\left(e_{i}\right)$ with concave $\phi, E U_{i}=P_{i}[\phi(v)-\phi(0)]+\phi(0)-C\left(e_{i}\right)$. Normalizing $\phi(0)$ to zero, we can simply replace $v$ with $\phi(v)$. A corollary is that if our agents were loss averse around fixed reference points they would behave qualitatively as in the standard model, as such loss aversion can be represented by a risk-averse concave utility function. Thus the endogeneity of the reference points is crucial to our later results with desert.

\subsection{Fair Tournaments with Desert}

When the tournament is fair, $k=0$ so $P_{i}=\widetilde{P}_{i}$ and the agents are identical. Using (1) we get: ${ }^{12}$

$$
E U_{i}\left(e_{i}, e_{j}\right)=v P_{i}-v \lambda P_{i}\left(1-P_{i}\right)-C\left(e_{i}\right)
$$

As $\lambda>0$ and $P_{i} \in(0,1)$, expected desert payoffs are always strictly negative. Letting

$$
\Omega_{i}\left(e_{i}-e_{j}\right) \equiv P_{i}\left(1-P_{i}\right)=Q\left(e_{i}-e_{j}\right)\left(1-Q\left(e_{i}-e_{j}\right)\right)
$$

we call the expression $v \lambda \Omega_{i}\left(e_{i}-e_{j}\right)$, which is strictly positive, agent $i$ 's desert deficit. From (5) we can see that the desert deficit is a concave function of $P_{i}$ that is maximized at $P_{i}=\frac{1}{2}$, i.e.,

${ }^{11} \overline{\text { Formally, suppose } e_{i}^{*}>e_{j}^{*} \geq 0 . e_{i}^{*}>0 \Rightarrow v q}\left(e_{i}^{*}-e_{j}^{*}+k\right)=C^{\prime}\left(e_{i}^{*}\right)$. But then $v q\left(e_{i}^{*}-e_{j}^{*}+k\right)>C^{\prime}\left(e_{j}^{*}\right)$, so $j$ has a strict incentive to work harder. The argument is the same when $e_{j}^{*}>e_{i}^{*} \geq 0$. Note that this argument does not depend on the assumption that $v q(k)>C^{\prime}(0)$.

${ }^{12}$ Although we have assumed preferences are kinked at the reference point, an unkinked quadratic loss function with $D_{i}(x)=g x^{2}$ for $x>0, D_{i}(x)=-l(-x)^{2}$ for $x \leq 0$ and $l=-g$ would give $E U_{i}=v P_{i}-v^{2} l P_{i}\left(1-P_{i}\right)-C\left(e_{i}\right)$, so qualitatively behavior would be unchanged. 
when $e_{i}=e_{j}$. It follows that the desert deficit is strictly quasi-concave in the effort difference and symmetric about zero.

Lemma 1 Each agent's desert deficit is given by the function $v \lambda \Omega_{i}\left(e_{i}-e_{j}\right)$ where $\Omega_{i}(x) \equiv$ $Q(x)(1-Q(x)) . \Omega_{i}(x)$ is (i) strictly positive and strictly quasi-concave for all $x$; (ii) maximized at $x=0$ where $P_{i}=\frac{1}{2}$; and (iii) symmetric about zero.

Proof. See Appendix.

Intuitively, when agents exert equal efforts and thus have equal chances of winning, both the winner and loser end up far from their common reference point. As one increases her effort above the other and so the chances of winning become less equal, the expected payoff of the favorite and the underdog become less equal, and it becomes more likely that the favorite wins. Thus, the average departure between agents' monetary payoffs and their reference points falls, reducing the scope for both desert losses and desert elation or guilt. Since losses loom larger than any elation by assumption, the overall desert deficit falls for both agents.

Using (4), (2) and (3), whenever efforts are strictly positive the first order conditions (FOCs) are:

$$
\begin{aligned}
& \frac{\partial E U_{i}}{\partial e_{i}}=v q\left(e_{i}-e_{j}\right)-v \lambda\left[\left(1-2 P_{i}\right) q\left(e_{i}-e_{j}\right)\right]-C^{\prime}\left(e_{i}\right)=0 \\
& \frac{\partial E U_{j}}{\partial e_{j}}=v q\left(e_{i}-e_{j}\right)-v \lambda\left[\left(1-2 P_{j}\right) q\left(e_{i}-e_{j}\right)\right]-C^{\prime}\left(e_{j}\right)=0
\end{aligned}
$$

while the second order conditions (SOCs) are:

$$
\begin{aligned}
\frac{\partial^{2} E U_{i}}{\partial\left(e_{i}\right)^{2}} & =v \frac{\partial q\left(e_{i}-e_{j}\right)}{\partial e_{i}}-v \lambda\left[\left(1-2 P_{i}\right) \frac{\partial q\left(e_{i}-e_{j}\right)}{\partial e_{i}}-2\left[q\left(e_{i}-e_{j}\right)\right]^{2}\right]-C^{\prime \prime}\left(e_{i}\right) \leq 0 \\
\frac{\partial^{2} E U_{j}}{\partial\left(e_{j}\right)^{2}} & =v \frac{-\partial q\left(e_{i}-e_{j}\right)}{\partial e_{i}}-v \lambda\left[\left(1-2 P_{j}\right) \frac{-\partial q\left(e_{i}-e_{j}\right)}{\partial e_{i}}-2\left[q\left(e_{i}-e_{j}\right)\right]^{2}\right]-C^{\prime \prime}\left(e_{j}\right) \leq 0 .
\end{aligned}
$$

\subsubsection{Symmetric Equilibria in Fair Tournaments}

The following summarizes properties of any symmetric desert equilibrium in a fair tournament.

\section{Proposition 2}

Any symmetric desert equilibrium must be the same as without desert.

For $\lambda \in\left[\frac{C^{\prime \prime}\left(C^{\prime-1}(v q(0))\right)}{4 v[q(0)]^{2}}, \frac{C^{\prime \prime}\left(C^{\prime-1}(v q(0))\right)}{2 v[q(0)]^{2}}\right]$, such a symmetric desert equilibrium will be asymptotically unstable.

For $\lambda>\frac{C^{\prime \prime}\left(C^{\prime-1}(v q(0))\right)}{2 v[q(0)]^{2}}$, i.e., for sufficiently strong desert, such a symmetric desert equilibrium cannot exist (as the second order conditions will be violated).

Proof. See Appendix. 
The result in Proposition 2 that in a symmetric desert equilibrium effort is the same as without desert is driven by the fact that at such a symmetric desert equilibrium, each agent has an equal chance of winning. As a result, $\Omega_{i}$ is maximized, and so from Lemma 1 the desert deficit is at its strongest. Because $\Omega_{i}$ is at an extremum, the effect of desert on marginal incentives is zero. The result should be contrasted with Grund and Sliwka's (2005) finding that in tournaments inequity-averse agents put in more effort in equilibrium. Grund and Sliwka's agents care about the equity of outcomes irrespective of effort levels and hence don't care whether outcomes were deserved or not. Receiving more than the rival induces compassion and receiving less gives rise to envy. Agents want to work harder to avoid envy and less hard to avoid compassion, and because envy is assumed to be a stronger emotion than compassion, the agents work harder in a symmetric equilibrium.

Around a symmetric desert equilibrium, if $e_{j}$ goes up slightly, $P_{i}$, and hence $\Omega_{i}$, falls. Thus, agent $i$ 's incentive to exert effort is reduced compared to the no desert case. Increasing effort raises the desert deficit by making the expected winnings more symmetrical, and so the reaction functions (RFs) become strictly downwards sloping rather than flat as in the no desert case. ${ }^{13}$ With sufficiently strong desert, the RFs become sufficiently downwards sloping that in $\left(e_{i}, e_{j}\right)$ space, $\mathrm{RF}_{j}$ crosses $\mathrm{RF}_{i}$ from above, and so any symmetric desert equilibrium becomes unstable (in the absence of desert stability is automatic). For very strong desert, the objective function becomes locally convex around the no desert symmetric equilibrium as the agents have too strong an incentive to create an asymmetry in order to reduce the large desert deficit, so the SOCs no longer hold and there is no symmetric desert equilibrium. ${ }^{14}$

\subsubsection{Asymmetric Equilibria in Fair Tournaments}

The finding that when we introduce desert, the symmetric equilibrium might be unstable, or indeed no longer exist at all, leads one to ask whether asymmetric equilibria are possible with desert. We saw above that without desert asymmetric equilibria are impossible. Furthermore, introducing inequity aversion with identical agents does not alter this basic finding, as discovered by Grund and Sliwka (2005). In this section, we investigate whether in a fair tournament asymmetric equilibria can arise with desert. We start by showing that for sufficiently weak desert concerns, there can be no asymmetric equilibrium.

\footnotetext{
${ }^{13}$ This means that, by contrast to the no desert case, if $j$ can precommit to a level of effort before $i$ chooses her effort, $j$ will have a local strategic incentive to choose effort above the desert equilibrium level. See Gill and Stone (2006, p. 12).

${ }^{14}$ Similarly to the case without desert, the need for global optimality may rule out a symmetric desert equilibrium even if the local SOCs are satisfied. One might wonder whether this might make it impossible for unstable symmetric desert equilibria to exist, but we can show that for sufficiently high noise or convex costs, stable and unstable symmetric desert equilibria can indeed exist (see Appendix B, part (i) in Gill and Stone, 2006).
} 
Proposition 3 For strictly positive $\lambda$ close enough to zero, no asymmetric desert equilibrium can exist.

Proof. See Appendix.

Next we show that for sufficiently strong desert concerns, asymmetric equilibria can indeed exist. We prove the existence of equilibria in which one agent works hard while the other slacks off completely, but, depending on $\lambda$, the shape of the noise distribution and the cost of effort function, less extreme interior asymmetric equilibria may also exist. Since the two agents are identical, they will have identical reaction functions. Let $e^{*}\left(e_{j}, \lambda\right)$ denote agent $i$ 's reaction function or global optimum given $e_{j}$ and $\lambda .^{15}$ Let $e^{*}(0, \lambda)$ denote the best response to an opponent exerting zero effort (throughout, if more than one exists, we take $e^{*}(0, \lambda)$ to refer to the lowest one).

We start by showing that $e^{*}(0, \lambda)>0$. Because we have assumed $v q(0)>C^{\prime}(0)$, even without desert the best response to no effort is strictly positive. Desert considerations simply increase the incentive to work when the rival slacks off, as doing so reduces the expected desert deficit. As $\lambda$ rises, the desert deficit gets stronger for any difference in the efforts, so the agent has a stronger incentive to push effort up to reduce the desert deficit, i.e., $e^{*}(0, \lambda)$ goes up.

Lemma 2 (i) $e^{*}(0, \lambda)>0$; (ii) $e^{*}(0, \lambda)$ is strictly increasing in $\lambda$; and (iii) $e^{*}(0, \lambda)$ is unbounded above as $\lambda$ rises.

\section{Proof. See Appendix.}

By raising $\lambda$ sufficiently, we can raise $e^{*}(0, \lambda)$ so high and make desert considerations so important that in response to $e^{*}(0, \lambda)>0$ the rival wants to set zero effort to reduce the desert deficit as much as possible (without incurring the huge cost of working harder than her rival), so we get asymmetric equilibria. Asymptotic stability follows in non-pathological cases. ${ }^{16}$

Proposition 4 For sufficiently large $\lambda$ : (i) there exist two asymmetric desert equilibria in each of which one agent exerts strictly positive effort $e^{*}(0, \lambda)>0$ and the other agent exerts zero effort as the unique best response; and (ii) such equilibria are asymptotically stable, so long as $e^{*}\left(e_{j}, \lambda\right)$ changes smoothly in $e_{j}$ at $e_{j}=0$.

${ }_{15} \overline{v P_{i} \text { and }-v \lambda P_{i}\left(1-P_{i}\right) \text { are bounded, while } C^{\prime \prime}}\left(e_{i}\right)>0$ implies that $C\left(e_{i}\right)$ is unbounded. Thus, given the continuity of $P_{i}$ and $C\left(e_{i}\right)$, a global optimum must exist, as $i$ will not wish to raise $e_{i}$ indefinitely. In general, more than one might exist.

${ }^{16}$ Cornes and Hartley (2003) introduce loss aversion into a Tullock-style rent-seeking contest. They focus on symmetric equilibria (which always exist), but note that asymmetric equilibria are also possible. Chowdhury and Sheremeta (2009) find asymmetric equilibria in a symmetric Tullock contest with spillovers. Stone (2006) finds that agents with self-image concerns may play an asymmetric equilibrium to avoid revealing too much information to themselves about their own ability. 
Proof. See Appendix.

Intuitively, in an asymmetric equilibrium in which $i$ is exerting zero effort and $j$ is exerting high effort $e^{*}(0, \lambda), j$ is more likely to win and feels that such a win is deserved while $i$ is more likely to lose but feels that such a loss is deserved. If $j$ lowers her effort or $i$ increases hers then on average the departure between monetary payoffs and agents' reference points will increase, increasing the desert deficit (see Lemma 1). Thus, agents have an incentive not to reduce the difference in their efforts. For sufficiently large $\lambda$ this force deters $i$ from increasing her effort above zero, even if doing so would increase her probability of winning sufficiently for the increase in expected monetary payoff to cover the increase in her effort costs. Given agent $i$ exerts zero effort, $j$ will increase her effort up until the point at which the marginal disutility of effort overwhelms the resulting marginal reduction of the desert deficit and marginal increase in her expected monetary payoff.

Of course, the existence of such asymmetric equilibria raises the question of how the players could coordinate on one of them. Exiting from the strict confines of the game at hand, some form of pre-play communication might aid coordination, or, with repeated play, a tâtonnement adjustment process could lead to one of the equilibria (Proposition 4 tells us that the equilibria are stable to such a process once they are reached). Note also that if the game was played twice, one equilibrium of the repeated game would be for the agents to play an asymmetric equilibrium in each period, but reversing the role of the hard worker and slacker across the periods. In the absence of discounting, the payoffs would become symmetric across the two periods. Again, pre-play communication could help coordinate on the roles.

\subsection{Unfair Tournaments with Desert}

We now consider unfair tournaments with desert, i.e., $k>0$ and $\lambda>0$. In particular we focus on symmetric equilibria: Section 4 considers asymmetric equilibria in a linear environment. ${ }^{17}$

Proposition 5 There can be no interior symmetric desert equilibrium. When the advantage $k>0$ is felt to be sufficiently deserved, i.e., $\theta$ is sufficiently close to 1, nor can there be a corner symmetric desert equilibrium in which both agents exert zero effort.

Proof. See Appendix.

In an unfair tournament, interior symmetric desert equilibria cannot arise, as when efforts are equal the advantaged agent always has a higher marginal incentive to increase effort. The

\footnotetext{
${ }^{17}$ Without solving for equilibrium, Ederer \& Fehr (2006, Sect. 2.2.2) briefly consider advantaged agents with loss aversion around the expected payoff (which is equivalent to the special case of $\theta=1$ in our model) in the context of feedback in a two-stage tournament with normally distributed noise.
} 
disadvantaged agent is more likely to lose, and so suffers more in expectation from increasing her deserved reference point $v \widetilde{P}_{j}$; at the same time, the disadvantaged agent starts with a (weakly) lower reference point, so losing is less painful and hence the agent benefits less in expectation from increasing her winning probability. This result, which holds however much the advantage is felt to be deserved (i.e., for any $\theta \in[0,1]$ ), should be contrasted with our findings for the no desert case, where any equilibrium is symmetric and interior, and fair tournaments with desert, where interior symmetric desert equilibria can arise when desert preferences are not too strong.

However, for low enough $\theta$ we cannot in general rule out a symmetric equilibrium where neither agent exerts any effort. Comparing to Propositions 1 and 2, such a zero-effort equilibrium cannot arise in the absence of either desert or an advantage. Suppose for instance that $q$ is unimodal. Then $q(\theta k)>q(k)$ for $\theta<1$, so increasing effort above zero increases the advantaged agent's deserved reference point faster than her expected monetary payoff. It is therefore possible for her expected desert payoff to go down given losing becomes more painful, and for this effect to outweigh the incentive to exert effort in the absence of desert.

\section{Linear Example}

In this section, we introduce a linear example to aid the understanding of the general results above and to analyze asymmetric equilibria of unfair tournaments. The results help to explain puzzling experimental results from unfair tournaments, and suggest a way to distinguish experimentally our theory of desert from pure disappointment aversion. Finally, we use the linear setup to illustrate that asymmetric equilibria can arise even when the uncertainty effect cannot.

\subsection{The Example}

In the example, we assume that noise is uniformly distributed, so winning probabilities are linear in the difference in the agents' efforts, which together with quadratic effort costs gives linear reaction functions.

$$
\text { Assumptions: } \quad \begin{cases}\text { (a) } & \eta \backsim U[-\gamma, \gamma] \\ \text { (b) } & e_{i} \leq \gamma-k \\ \text { (c) } & C\left(e_{i}\right)=\frac{c e_{i}^{2}}{2} \\ \text { (d) } & v \leq \frac{4 \gamma^{2} c(\gamma-k)}{2 \gamma+\lambda(2 \gamma-(1-\theta) k)}\end{cases}
$$


Under (a), and given $e_{i}-e_{j} \leq \gamma-k$ and $e_{j}-e_{i} \leq \gamma-k$ by the upper bound on efforts (b):

$$
\begin{aligned}
P_{i}\left(e_{i}, e_{j}\right) & =Q\left(e_{i}-e_{j}+k\right)=\frac{e_{i}-e_{j}+k+\gamma}{2 \gamma} \in[0,1] ; \\
\widetilde{P}_{i}\left(e_{i}, e_{j}\right) & =Q\left(e_{i}-e_{j}+\theta k\right)=\frac{e_{i}-e_{j}+\theta k+\gamma}{2 \gamma} \in[0,1] ; \\
q\left(e_{i}-e_{j}+k\right) & =q\left(e_{i}-e_{j}+\theta k\right)=\frac{1}{2 \gamma}>0 ;
\end{aligned}
$$

so the model is consistent with our general framework over the permitted range of efforts. ${ }^{18}$ The restriction (b) on efforts could for instance be interpreted as a time restriction. The $\gamma$ parameter is a measure of noise: the greater the noise, the lower the marginal effect of effort on the probability of winning. (d) ensures global concavity of the objective functions, and also ensures that (b) does not bind on any agent's choice. The following proposition is illustrated in Figures 1 and 2 .

\section{Proposition 6}

(i) If $\lambda<\frac{2 \gamma^{2} c}{2 v+\gamma c(1+\theta) k}$, there is a unique, interior and stable desert equilibrium, which is symmetric in a fair tournament and in which the advantaged agent works harder in an unfair tournament.

(ii) If $\lambda \in\left[\frac{2 \gamma^{2} c}{2 v+\gamma c(1+\theta) k}, \frac{2 \gamma^{2} c}{2 v-\gamma c(1+\theta) k}\right)$, which can only occur in an unfair tournament, there is a unique and stable desert equilibrium, in which the advantaged agent exerts strictly positive effort and the disadvantaged agent exerts zero effort. ${ }^{19}$

In both cases, when the tournament is unfair, the difference between the efforts rises as the advantage $k$ gets bigger, the extent to which the advantage is felt to be deserved $\theta$ increases or desert preferences $\lambda$ become stronger.

Proof. See Appendix.

The equilibria should be compared to those in the absence of desert (Proposition 1), in which the advantaged agent exerts the same effort as the disadvantaged agent. Section 3.3 explained why, in general and starting from symmetry, the advantaged agent has a stronger marginal incentive to exert effort in the presence of desert. As is intuitive, the effort difference induced by the advantage increases in the size of the advantage, the extent to which the advantage is

${ }^{18}$ No standard underlying noise function that we are aware of would give $\eta$ uniformly distributed. However, we have chosen a uniform distribution here for its analytical and pedagogical convenience. We can also think of the noise as arising from the measurement of the difference in efforts rather than of each agent's separate effort level.

${ }^{19}$ In the special case where $2 v \leq \gamma c(1+\theta) k$, the upper bound does not apply. Gill and Stone (2006, pp. 16-17) consider the case where $2 v>\gamma c(1+\theta) k$ and $\lambda>\frac{2 \gamma^{2} c}{2 v-\gamma c(1+\theta) k}$ for fair tournaments, which gives stable asymmetric equilibria. In an unfair tournament, it is possible that in this case the disadvantaged agents works hardest, either in an unstable interior equilibrium or in one in which the advantaged agent exerts zero effort. 
felt to be deserved and the strength of desert preferences. The bigger the advantage, the more likely the advantaged agent is to win for any given effort pair, so an increase in the deserved reference point $v \widetilde{P}_{i}$ from working harder is less painful in expectation. The greater the extent to which the advantage is felt to be deserved, the bigger is the reference point, which means that losing becomes more painful so the advantaged agent has a greater incentive to exert effort to reduce the chance of losing.
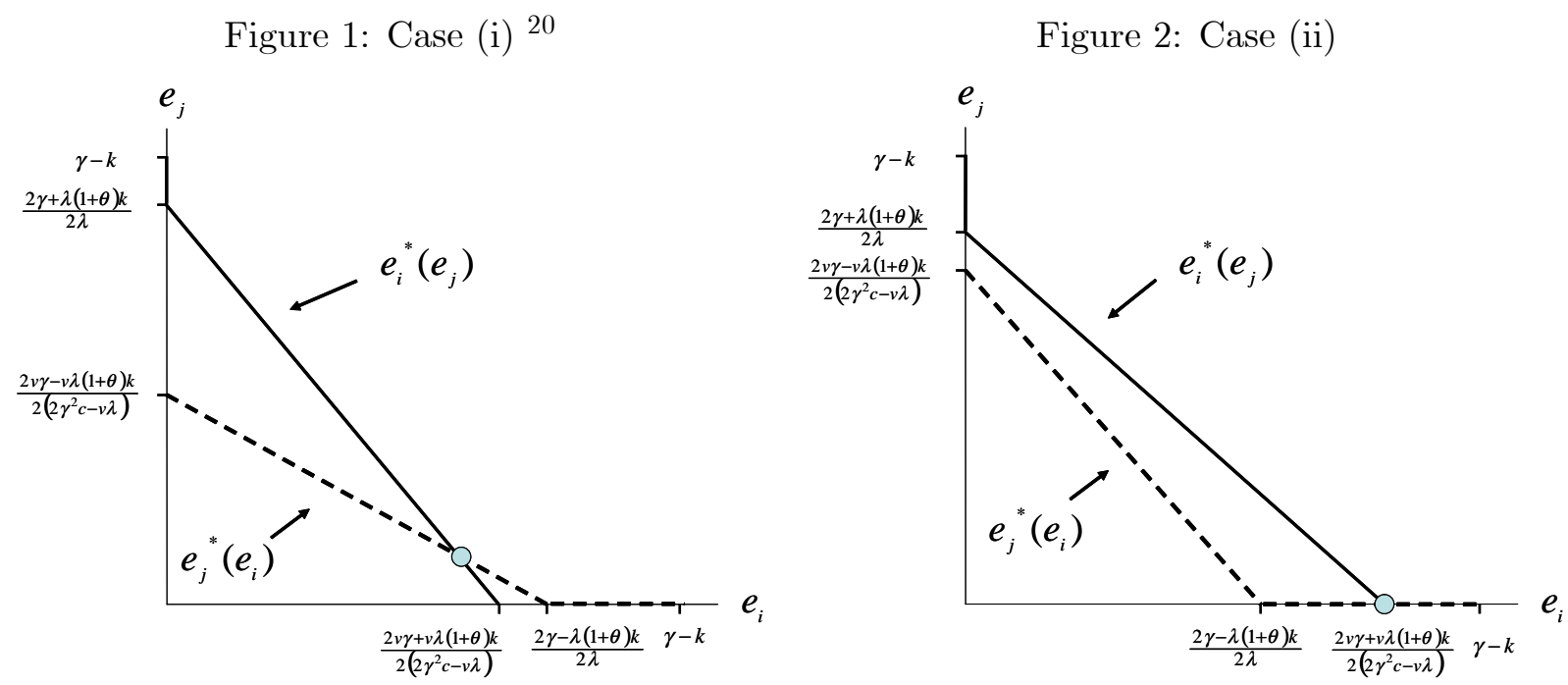

\subsection{Experimental Evidence and Testing}

Our results may help to explain two puzzling results from tournaments in the laboratory and suggest an experiment to distinguish desert from disappointment aversion (see Gill and Prowse, 2009 , for an experimental test of disappointment aversion in a sequential-move tournament).

First, the experimental evidence on fair tournaments has found excess variability in mean effort choices (see for instance the classic paper by Bull et al., 1987, and, more recently, van Dijk et al., 2001). This excess variability is consistent with the agents attempting to coordinate on an asymmetric equilibrium, as predicted by Proposition 4 for sufficiently strong desert. ${ }^{21}$ Interestingly, in a setting with multiple prizes where the theoretical prediction was for the agents to all exert maximal effort, Harbring and Irlenbusch (2003) report commonly finding highly asymmetric behavior, with some agents in a particular group putting in maximal effort and the others exerting very low effort, while Sheremeta (2009) finds evidence of 'drop-outs' in a symmetric Tullock-style rent seeking contest.

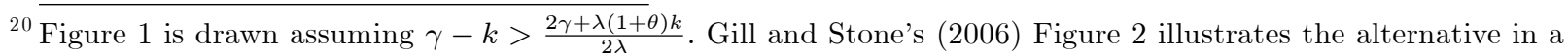
fair tournament: the unfair tournament is a natural generalization.

${ }^{21}$ The experimental evidence on fair tournaments tends to report only the mean of effort choices across pairs of players (and not the variance between a pair's effort choices). 
Second, Schotter and Weigelt (1992) found that in unfair tournaments advantaged agents tend to work harder than their disadvantaged counterparts. These results are contrary to standard theory, under which the equilibrium remains symmetric, but consistent with the results in Propositions 5 and 6.

Finally, the results suggest that we may distinguish experimentally between desert concerns and disappointment aversion by manipulating the extent to which any advantage is felt to be deserved. For example, we could introduce a prior stage in which the advantage is either arbitrarily allocated or allocated in an effort-sensitive manner according to performance in a task. If agents are disappointment averse (i.e., they are loss averse around their expected monetary payoff), they will always act like agents with desert preferences who feel the advantage is fully deserved, i.e., with $\theta=1$, even if the advantage was allocated arbitrarily rather than meritocratically. Thus, unlike standard agents, advantaged disappointment averse agents will work harder than their disadvantaged counterparts, but unlike desert-concerned agents they will not be sensitive to the source of the advantage. Accordingly, if only disappointment aversion is motivating agents, the method of allocating the advantage will not affect tournament outcomes.

\subsection{The Uncertainty Effect}

Gneezy et al. (2006) recently provided experimental evidence for what they call the uncertainty effect, whereby a lottery is valued at less than the lottery's lowest possible outcome. The uncertainty effect is a breach of monotonicity as stochastically dominating gambles are rejected. Gneezy et al. note that certain models of disappointment aversion (e.g., Bell, 1985) can explain such preferences. When $\lambda>1$ and $P_{i}$ is sufficiently small, our model also predicts the uncertainty effect. This follows as in a fair tournament (or an unfair one with $\theta=1$ ), for a given level of effort an agent prefers a zero chance of winning to the lottery implied by $P_{i}>0$ if and only if

$$
0 \geq v P_{i}-v \lambda P_{i}\left(1-P_{i}\right) \Leftrightarrow P_{i} \leq \frac{\lambda-1}{\lambda}
$$

Kőszegi and Rabin's model of reference-dependence with a choice-acclimating reference point (2007, Section IV) shares the same feature, and the authors claim that $\lambda>1$ is calibrationally plausible (our parameter $\lambda$ is equivalent to $\eta(1-\lambda)$ in their model). Nonetheless, we show by example that asymmetric equilibria can exist and be unique in a fair tournament when $\lambda \leq 1$ so the uncertainty effect cannot arise. Take a simple linear example with $\eta \sim U[-1,1]$, so the probability of winning is $P_{i}=\frac{e_{i}-e_{j}+1}{2}$ given an upper bound of 1 on effort (which ensures that $q=\frac{1}{2}>0$ for all permitted efforts), $k=0, v=1$ and $C\left(e_{i}\right)=\frac{c e_{i}^{2}}{2}+\frac{2}{5} e_{i}$. 
Claim 1 Suppose $\lambda=\frac{1}{2}$. When $c=\frac{2}{5}$, there exist two asymmetric equilibria, in each of which one agent exerts zero effort while the other sets $e_{i}=\frac{2}{3}$. There is also a symmetric equilibrium, but it is unstable. When $c=\frac{1}{5}$, we get uniqueness of the asymmetric equilibrium (up to reversing the role of the agents), with one agent setting zero effort and the other setting effort at the upper bound of 1 .

Proof. See Appendix.

\section{Applications in Fair Tournaments}

We now turn to two applications of our theory of desert in fair tournaments, the first using asymmetric equilibria and the second symmetric ones.

First, we analyze competition for status in the presence of desert concerns. We show that when desert is strong enough that agents play an asymmetric equilibrium, competition for status can dominate a random allocation of status / rank, even though effort is socially wasteful. Thus desert considerations can provide a psychological basis for allowing agents to compete for a fixed supply of status.

Second, we consider an employer's choice of relative performance compensation scheme. We show that when desert is not too strong, so employees play a symmetric equilibrium, an employer will choose a tournament over relative performance pay linear in the difference in the employees' outputs if output noise is sufficiently fat-tailed. Desert considerations thus provide a new explanation for why employers might choose to use tournament-style incentive schemes.

\subsection{Status Competition}

Agents often compete for status within a group, where an agent's status is defined as her ordinal rank in the group. Within a group the supply of rank is fixed, which is why a number of authors have considered competition for status to be a socially wasteful zero-sum game. Recent notable examples of such a perspective are presented by Hopkins and Kornienko (2004, pp. 1091-1092) and Frank (2005, p. 137), who consider agents competing for status by spending on positional goods.

We can interpret our tournament as a competition for status. Agents care about their relative rank in the distribution of $\psi$, with $\psi_{i}$ distributed as before, and they value a higher rank at $v$. Much of the literature has focused on status as determined by spending on positional goods, and we can think of $\psi$ as such spending, where agents exert effort to increase the budget they can spend on such goods. Our model can also incorporate many other types of status 
concerns. For example, we can think of winning the tournament as being allocated a position of higher importance in an organization.

In the absence of desert concerns, the zero-sum nature of the final status allocation does indeed lead to the conclusion that competition over status is socially wasteful. In the unique equilibrium of the game, the agents exert the same level of effort so each has an equal opportunity of winning and losing. Thus, the agents would be better off if they could somehow enforce an equal reduction in their efforts, since this would reduce wasteful expenditure on effort while leaving the winning probabilities unchanged. It follows that both agents would be better off if competition was banned and social rank was instead determined randomly.

The conclusion that banning competition is good for welfare may be undermined when agents have desert concerns. ${ }^{22}$ This is true whenever asymmetric equilibria can arise. An asymmetric equilibrium has the feature that, more often than not, it will allocate the higher status to the agent who exerted higher effort and therefore deserves it more and the lower status to the agent who deserves it less. By contrast, when status is allocated randomly, as when competition is banned or when competing agents exert identical efforts, the outcome is less satisfactory in desert terms: although neither agent is more deserving than the other, one agent is always deemed the winner and the other the loser at the end of the tournament. Thus, on average, the discrepancy between what an agent receives ex post and what she deserves given agents' efforts is larger than in any asymmetric equilibrium - as shown in Lemma 1, the desert deficit is always lower the more unequal the winning probabilities. When competition is not permitted, the higher desert deficit can overwhelm the benefit of lower effort, compared to an asymmetric equilibrium under competition. Thus desert considerations can provide a psychological basis for preferring competition, even in what appears to be a zero-sum game such as a competition for status. Gill and Stone (2006, pp. 18-20) provide technical details.

\subsection{Relative Performance Pay Schemes}

Firms commonly use tournament-type incentive schemes to motivate staff. ${ }^{23}$ In this section, we analyze how desert preferences impact on the design of relative performance incentive schemes, thus contributing to the literature which considers when firms might choose to implement tournaments and to the rapidly growing behavioral contract literature. ${ }^{24}$ Throughout, we assume

${ }_{22}$ Of course, the conclusion is also undermined if positive externalities from effort are sufficiently strong. We show that even absent any such externalities, desert considerations can reverse the standard argument.

${ }^{23}$ For some recent evidence see DeVaro, 2006. See also Conyon et al., 2001, Prendergast, 1999, and the references therein.

${ }^{24}$ For a survey of the literature on behavioral contract theory more generally, see Englmaier (2005). Bartling and von Siemens (2004), Itoh (2004) and Rey Biel (2008) all analyze how inequity aversion over monetary payoffs net of effort costs impact on compensation design in very simple frameworks with binary effort choices. 
that from the perspective of firms efforts are either unobservable or unverifiable, so compensation must be based on output.

A number of papers have compared tournaments to individual performance-based compensation (such as piece rates). A first strand of literature emphasizes that tournaments iron out common output shocks that are unobservable to the employer. In the presence of risk aversion they will thus be preferred if the variance of the common shock is sufficiently large (see for instance Green and Stokey, 1983). However, tournaments are not the only incentive scheme which iron out such shocks: other schemes which base payments on the relative output of workers can also do the trick. A second strand of literature points out that tournaments fix total payments in advance, so employers do not have the incentive to under-report performance that occurs with individual performance schemes (see Malcomson, 1984). Yet again, however, not just tournaments but any relative performance scheme with fixed total payments solves the problem.

We compare how our tournament (which is the simplest rank-based incentive scheme) fares relative to the simplest incentive scheme which takes the magnitude of the difference in outputs into account, namely pay linear in the output difference of the workers. We call the latter linear relative performance pay (LRPP). Both schemes iron out common additive output shocks ${ }^{25}$ and entail a fixed total wage bill. In the absence of desert, we find that the employer is indifferent between the two schemes, while in the presence of desert considerations the shape of the noise distribution has a fundamental impact on an employer's choice between these two relative performance pay schemes. ${ }^{26}$

We assume that an employer is designing an incentive scheme for two identical workers with weak desert preferences. The employer is required to induce a total effort $\widehat{e}>0$. However, our results are robust to allowing the employer to choose productive effort to maximize profits (see Gill and Stone, 2006, Corollary 1, p. 24). Under the fair tournament, the employer chooses a fixed payment of $F_{T}$ to each worker and a prize $v$. For low enough $\lambda$, any desert equilibrium is symmetric by Proposition 3, and we assume that such a symmetric equilibrium exists. Under LRPP, the employer pays each worker a wage $w_{i}$ linear in the difference in their outputs. The employer chooses the strength of incentives $\alpha$ plus a fixed payment $F_{L R P P}$ :

$$
w_{i}=\alpha\left(\psi_{i}-\psi_{j}\right)+F_{L R P P}
$$

\footnotetext{
${ }^{25} \overline{\text { We abstract from such shocks in the analysis }}$ below, but introducing an additive common output shock does not alter any of our results.

${ }^{26}$ Nalebuff and Stiglitz (1983, pp. 36-37) analyzed LRPP, comparing it to the use of piece rates. The only existing comparison of tournaments and LRPP that we are aware of occurs in McLaughlin (1988, p.235), who claims to find that in a free entry model with risk aversion and normal noise, the tournament can induce greater effort.
} 
As in the fair tournament, under LRPP each worker's reference point is taken to be her expected monetary payoff, ${ }^{27}$ so:

$$
r_{i}=E\left[\alpha\left(\psi_{i}-\psi_{j}\right)+F_{L R P P}\right]=\alpha\left(e_{i}-e_{j}\right)+F_{L R P P}
$$

The employer must design the scheme to satisfy the workers' participation constraint. In particular each worker's expected utility must cover her outside option $\bar{U}$. For simplicity we assume that the workers face no ex post credit constraints ${ }^{28}$

Proposition 7 For $\lambda$ low enough that target effort $\widehat{e}$ is induced by a symmetric desert equilibrium in a fair tournament, wage costs are lower under the tournament than under linear relative performance pay if and only if $2 q(0) E[|\eta|] \geq 1$. In the absence of desert, the two schemes cost the same.

Proof. See Appendix.

In the absence of desert, the employer has to compensate the workers just for their cost of effort and outside option, so the two schemes have the same cost. With desert, the employer also has to compensate the workers for their expected desert losses. Remarkably, the comparison of the two payment schemes depends only on the shape of the noise distribution. In particular, it is independent of the size of the target $\widehat{e}$, and the strength of desert $\lambda$ (within the relevant range).

A higher $q(0)$ favors the tournament, as marginal incentives at the symmetric equilibrium are higher and so the prize required to induce a given level of effort is lower. In the absence of desert this would not make the tournament any cheaper, as the fixed payments would have to rise correspondingly to satisfy the participation constraint. However, in the presence of desert a lower prize lowers the desert deficit, as the workers' wages will be closer to their reference points on average, and so wage costs are lower. Under LRPP, on the other hand, $q(0)$ has no effect on marginal incentives.

Fatter tails as measured by $E[|\eta|]$ also favor the tournament. With fatter tails, $\eta$ is more likely to be far from its mean, and hence under LRPP the workers are more likely to receive wages far from their reference point, increasing the fixed payments necessary to compensate them for their expected desert losses. Under a tournament, however, the desert deficit depends only on the prize and the probability of winning, which are independent of $E[|\eta|]$ for a given $q(0)$ at the symmetric equilibrium.

\footnotetext{
${ }^{27} \mathrm{As}$ in the tournament, the reference point reflects the marginal impact of effort on the expected payment and is meritocratic.

${ }^{28}$ See Gill and Stone (2006, footnote 27) for a discussion of this assumption.
} 
For normally distributed noise, LRPP is always strictly cheaper whatever the variance. As the variance rises, $q(0)$ falls, while $E[|\eta|]$ rises in an exactly compensating fashion. However, under a fatter-tailed distribution the tournament can dominate, for example when noise is distributed according to the Student's t-distribution with $z \in(1,2)$ degrees of freedom. Gill and Stone (2006, pp. 24-26) provide the details and use an example based on the Student's tdistribution to show that choosing the right compensation scheme can have a significant impact on the wage bill.

We have found that wage costs are lower under a tournament than under LRPP if the distribution of noise is sufficiently fat-tailed, given that the required effort level is induced by a symmetric desert equilibrium in the tournament. Tournaments may also be preferred if the required effort can be induced by an asymmetric desert equilibrium or if the employer chooses to induce an asymmetric equilibrium when she selects effort optimally. Pushing the workers' effort levels apart lowers the desert deficit, which in turn lowers the fixed fee needed to satisfy the workers' participation constraint. Of course, this is counterbalanced by the fact that it is less efficient to make one worker take on the whole task: $C(\widehat{e})>2 C\left(\frac{\widehat{e}}{2}\right)$ by the convexity of costs. See Gill and Stone (2006, Section 5.2) for an analysis of this issue.

\section{Conclusion}

In this paper, we have merged the literatures on tournaments, equity and loss aversion in order to model the behavior of desert-motivated agents in competitive situations. In line with existing psychological and experimental evidence, our agents adopt a meritocratic notion of desert. Our model has allowed us to develop novel conclusions about the play of identical, advantaged and disadvantaged agents in tournaments. In doing so, we have contributed to the understanding of strategic behavior in the presence of endogenous reference points. Applying our model has also permitted us to generate insights into when competition for status might be socially beneficial and when employers might choose tournaments over other more continuous forms of relative performance pay.

Fruitful extensions to our framework might analyze situations with many agents and prizes or apply our concept of desert to other strategic settings such as bargaining and the provision of public goods. Empirical and experimental evidence could also be collected to test whether agents behave according to the theoretical predictions of our model and so do in fact act as if they care about receiving their 'just deserts'. 


\section{Appendix}

Proof of Lemma 1. $\Omega_{i}(x)>0$ given $Q(x) \in(0,1)$. Clearly $\Omega_{i}(x)$ is maximized when $Q=\frac{1}{2}$, so $x=0 . \Omega_{i}(x)$ is strictly quasi-concave in the effort difference, and hence in each agent's effort, since

$$
\frac{\partial \Omega_{i}\left(e_{i}-e_{j}\right)}{\partial e_{i}}=\frac{\partial \Omega_{i}\left(e_{i}-e_{j}\right)}{\partial\left(e_{i}-e_{j}\right)}=q\left(e_{i}-e_{j}\right)\left(1-2 Q\left(e_{i}-e_{j}\right)\right)
$$

which is strictly positive whenever $e_{i}<e_{j}$ since then $Q\left(e_{i}-e_{j}\right)<\frac{1}{2}$, strictly negative if $e_{i}>e_{j}$ and zero if $e_{i}=e_{j}$. Finally, note that $P_{i}=1-P_{j}$, so $P_{i}\left(1-P_{i}\right)=P_{j}\left(1-P_{j}\right)$, and the agents always face the same desert deficit, i.e., $\Omega_{i}(x)=\Omega_{j}(-x)$. By the symmetry of the agents, $\Omega_{j}(-x)=\Omega_{i}(-x)$. Putting these two facts together, we see that $\Omega_{i}\left(e_{i}-e_{j}\right)$ is symmetric about zero.

Proof of Proposition 2. Assuming symmetry, $e_{i}=e_{j}$ so $P_{i}=P_{j}=Q(0)=\frac{1}{2}$. Thus, $1-2 P_{i}=0$ and the desert terms in the derivatives in (6) drop out, so any symmetric equilibrium must be the same as without desert. The symmetry of $q(\eta)$ about zero implies that $\frac{\partial q(0)}{\partial e_{i}}=0$, so given symmetry, agent $i$ 's $\operatorname{SOC}(7)$ reduces to $\lambda \leq \frac{C^{\prime \prime}\left(C^{\prime-1}(v q(0))\right)}{2 v[q(0)]^{2}}$. Finally, we consider asymptotic stability (see Fudenberg and Tirole, 1991, pp. 23-25, for more on asymptotic stability and tâtonnement adjustment processes). Consider $i^{\prime} s$ reaction function (RF) when the symmetric equilibrium exists. On this RF, $d \frac{\partial E U_{i}}{\partial e_{i}}=\frac{\partial^{2} E U_{i}}{\partial\left(e_{i}\right)^{2}} d e_{i}^{*}+\frac{\partial^{2} E U_{i}}{\partial e_{j} \partial e_{i}} d e_{j}=0, s o \frac{d e_{i}^{*}}{d e_{j}}=-\frac{\frac{\partial^{2} E U_{i}}{\partial e_{j} \partial e_{i}}}{\frac{\partial^{2} E U_{i}}{\partial\left(e_{i}\right)^{2}}}$. The cross-derivative is:

$$
\frac{\partial^{2} E U_{i}}{\partial e_{j} \partial e_{i}}=v \frac{-\partial q\left(e_{i}-e_{j}\right)}{\partial e_{i}}-v \lambda\left[\left(1-2 P_{i}\right) \frac{-\partial q\left(e_{i}-e_{j}\right)}{\partial e_{i}}+2\left[q\left(e_{i}-e_{j}\right)\right]^{2}\right] .
$$

At a symmetric equilibrium $\frac{\partial q(0)}{\partial e_{i}}=0,1-2 P_{i}=0$ and the SOC must hold, so the slope of $i^{\prime} s$ $\mathrm{RF}$ is:

$$
\frac{d e_{i}^{*}}{d e_{j}}=\frac{-2 v \lambda[q(0)]^{2}}{C^{\prime \prime}\left(C^{\prime-1}(v q(0))\right)-2 v \lambda[q(0)]^{2}}<0
$$

The RFs are downwards sloping and have the same slope, so any symmetric equilibrium will be asymptotically stable if and only if:

$$
\frac{d e_{i}^{*}}{d e_{j}}>-1 \Leftrightarrow 4 v \lambda[q(0)]^{2}<C^{\prime \prime}\left(C^{\prime-1}(v q(0))\right)
$$

Note that if instead of having a well-behaved RF, agent $i^{\prime} s$ best response jumps discontinuously (because of multiple best responses at the symmetric equilibrium, e.g., because the SOC is satisfied with equality), clearly stability cannot hold. 
Proof of Proposition 3. Suppose that an asymmetric equilibrium $\left(e_{i}^{*}, e_{j}^{*}\right)$ exists with $e_{i}^{*}>e_{j}^{*}$. From the FOCs in (6), noting that $1-2 P_{j}=2 P_{i}-1$ and allowing for the possibility that $e_{j}^{*}=0$ :

$$
\frac{\partial E U_{i}}{\partial e_{i}}-\frac{\partial E U_{j}}{\partial e_{j}}=2 v \lambda\left[\left(2 P_{i}-1\right) q\left(e_{i}^{*}-e_{j}^{*}\right)\right]-C^{\prime}\left(e_{i}^{*}\right)+C^{\prime}\left(e_{j}^{*}\right) \geq 0 .
$$

Let $\bar{q} \equiv \sup q(\eta)$, which must exist as $q(\eta)$ is a density continuous on $(-\infty, \infty)$. Then $v \lambda\left[\left(2 P_{i}-1\right) q\left(e_{i}-e_{j}\right)\right] \leq v \lambda \bar{q}$, so $2 v \lambda \bar{q} \geq C^{\prime}\left(e_{i}^{*}\right)-C^{\prime}\left(e_{j}^{*}\right)$. Now $C^{\prime}\left(e_{i}^{*}\right)>C^{\prime}\left(e_{j}^{*}\right)$ and as $\lambda \rightarrow 0,2 v \lambda \bar{q} \rightarrow 0$. Thus as $\lambda \rightarrow 0, C^{\prime}\left(e_{i}^{*}\right)-C^{\prime}\left(e_{j}^{*}\right)$ goes to zero, so $e_{i}^{*}-e_{j}^{*}$ goes to zero. Thus from the FOC $e_{i}^{*}$ goes to $C^{\prime-1}(v q(0))>0$ so $e_{j}^{*}$ does so as well.

Let $e_{x}$ be "near" to $C^{\prime-1}(v q(0))$ iff

$$
\left|e_{x}-C^{\prime-1}(v q(0))\right| \leq \max \left\{\left|e_{i}^{*}-C^{\prime-1}(v q(0))\right|,\left|e_{j}^{*}-C^{\prime-1}(v q(0))\right|\right\} .
$$

We now consider the slope of the agents' reaction functions (RFs) for efforts near to $C^{\prime-1}(v q(0))$. The slope of $\mathrm{RF}_{i}$ is given by $-\frac{\frac{\partial^{2} E U_{i}}{\partial e_{j} \partial e_{i}}}{\frac{\partial^{2} E U_{i}}{\partial\left(e_{i}\right)^{2}}}$. As $\lambda \rightarrow 0,(14) \rightarrow 0$ and $(7) \rightarrow-C^{\prime \prime}\left(e_{i}\right)$ (remember $\frac{\partial q(0)}{\partial e_{i}}=0$ and $q^{\prime}$ is continuous) for efforts near to $C^{\prime-1}(v q(0))$, so the slope of the RFs go to zero. In $\left(e_{i}, e_{j}\right)$ space, $\mathrm{RF}_{i}$ tends to a vertical line while $\mathrm{RF}_{j}$ tends to a horizontal line. Thus there exists a $\delta>0$ such that for all $\lambda<\delta$, the RFs can only cross once near to $C^{\prime-1}(v q(0))$, so there must be a single equilibrium near to $C^{\prime-1}(v q(0))$. But by the symmetry of the problem there must be (at least) two asymmetric equilibria near to $C^{\prime-1}(v q(0))$, so we get contradiction.

Proof of Lemma 2. Note first that given $e_{j}=0, \Omega_{i}^{\prime}(0)=0$ at $e_{i}=0$ and $\Omega_{i}^{\prime}\left(e_{i}\right)<0$ for $e_{i}>0$ from Lemma 1 .

(i) Suppose $e^{*}(0, \lambda)=0 . \Omega_{i}^{\prime}(0)=0$ and by assumption $v q(0)>C^{\prime}(0)$. Thus, $\frac{\partial E U_{i}\left(e_{i}, 0, \lambda\right)}{\partial e_{i}}=$ $v q(0)-C^{\prime}(0)>0$ at $e_{i}=0$, so $i$ has a strict incentive to increase effort, a contradiction.

(ii) We suppose that for $\lambda_{2}>\lambda_{1}, e^{*}\left(0, \lambda_{2}\right) \leq e^{*}\left(0, \lambda_{1}\right)$ and find a contradiction.

Case (a): $e^{*}\left(0, \lambda_{2}\right)<e^{*}\left(0, \lambda_{1}\right)$. As $e^{*}(0, \lambda)>0$ and $\Omega_{i}^{\prime}\left(e_{i}\right)<0$ for $e_{i}>0, \Omega_{i}\left(e^{*}\left(0, \lambda_{1}\right)\right)-$ $\Omega_{i}\left(e^{*}\left(0, \lambda_{2}\right)\right)<0$. By definition of global optimality:

$\left[E U_{i}\left(e^{*}\left(0, \lambda_{1}\right), 0, \lambda_{1}\right)-E U_{i}\left(e^{*}\left(0, \lambda_{2}\right), 0, \lambda_{1}\right)\right]+\left[E U_{i}\left(e^{*}\left(0, \lambda_{2}\right), 0, \lambda_{2}\right)-E U_{i}\left(e^{*}\left(0, \lambda_{1}\right), 0, \lambda_{2}\right)\right] \geq 0$.

But:

$$
\begin{aligned}
& E U_{i}\left(e^{*}\left(0, \lambda_{1}\right), 0, \lambda_{1}\right)-E U_{i}\left(e^{*}\left(0, \lambda_{1}\right), 0, \lambda_{2}\right)=-\left(\lambda_{1}-\lambda_{2}\right) v \Omega_{i}\left(e^{*}\left(0, \lambda_{1}\right)\right) ; \\
& E U_{i}\left(e^{*}\left(0, \lambda_{2}\right), 0, \lambda_{2}\right)-E U_{i}\left(e^{*}\left(0, \lambda_{2}\right), 0, \lambda_{1}\right)=-\left(\lambda_{2}-\lambda_{1}\right) v \Omega_{i}\left(e^{*}\left(0, \lambda_{2}\right)\right) ;
\end{aligned}
$$


so we require that $\left(\lambda_{2}-\lambda_{1}\right) v\left[\Omega_{i}\left(e^{*}\left(0, \lambda_{1}\right)\right)-\Omega_{i}\left(e^{*}\left(0, \lambda_{2}\right)\right)\right] \geq 0$, a contradiction.

Case (b): $e^{*}\left(0, \lambda_{2}\right)=e^{*}\left(0, \lambda_{1}\right)$. Given $e^{*}\left(0, \lambda_{1}\right)>0$ the FOCs imply the following which, together with $\Omega_{i}^{\prime}\left(e^{*}\left(0, \lambda_{1}\right)\right)<0$, contradicts $\lambda_{2}>\lambda_{1}$ :

$$
v q\left(e^{*}\left(0, \lambda_{1}\right)\right)-v \lambda_{2} \Omega_{i}^{\prime}\left(e^{*}\left(0, \lambda_{1}\right)\right)=v q\left(e^{*}\left(0, \lambda_{1}\right)\right)-v \lambda_{1} \Omega_{i}^{\prime}\left(e^{*}\left(0, \lambda_{1}\right)\right) .
$$

(iii) Given $e_{j}=0$ and $e_{i}>0, \Omega_{i}^{\prime}\left(e_{i}\right)<0$. Thus for any $x>0$, we can find a $\lambda>0$ such that for $\forall e_{i} \in(0, x]$ :

$$
\frac{\partial E U_{i}\left(e_{i}, 0, \lambda\right)}{\partial e_{i}}=v q\left(e_{i}\right)-v \lambda \Omega_{i}^{\prime}\left(e_{i}\right)-C^{\prime}\left(e_{i}\right)>0,
$$

so $e^{*}(0, \lambda)>x$.

Proof of Proposition 4. We start by using a proof by contradiction in two parts to show that for $e_{j}>0$, the difference between an agent's global optimum effort $e^{*}\left(e_{j}, \lambda\right)$ and her rival's effort $e_{j}$ is always less than the best response to zero effort, i.e.,

$$
e^{*}\left(e_{j}, \lambda\right)-e_{j}<e^{*}(0, \lambda) \text {. }
$$

Case (1): suppose that $e^{*}\left(e_{j}, \lambda\right)-e_{j}>e^{*}(0, \lambda)$. By definition of global optimality:

$\left[E U_{i}\left(e^{*}(0, \lambda), 0, \lambda\right)-E U_{i}\left(e^{*}\left(e_{j}, \lambda\right)-e_{j}, 0, \lambda\right)\right]+\left[E U_{i}\left(e^{*}\left(e_{j}, \lambda\right), e_{j}, \lambda\right)-E U_{i}\left(e^{*}(0, \lambda)+e_{j}, e_{j}, \lambda\right)\right] \geq 0$.

But:

$$
\begin{aligned}
E U_{i}\left(e^{*}(0, \lambda), 0, \lambda\right)-E U_{i}\left(e^{*}(0, \lambda)+e_{j}, e_{j}, \lambda\right) & =-C\left(e^{*}(0, \lambda)\right)+C\left(e^{*}(0, \lambda)+e_{j}\right)>0 ; \\
E U_{i}\left(e^{*}\left(e_{j}, \lambda\right), e_{j}, \lambda\right)-E U_{i}\left(e^{*}\left(e_{j}, \lambda\right)-e_{j}, 0, \lambda\right) & =-C\left(e^{*}\left(e_{j}, \lambda\right)\right)+C\left(e^{*}\left(e_{j}, \lambda\right)-e_{j}\right)<0 ;
\end{aligned}
$$

so we require that $C\left(e^{*}(0, \lambda)+e_{j}\right)-C\left(e^{*}(0, \lambda)\right) \geq C\left(e^{*}\left(e_{j}, \lambda\right)\right)-C\left(e^{*}\left(e_{j}, \lambda\right)-e_{j}\right)$. Because $C^{\prime \prime}\left(e_{i}\right)>0$, an increase in effort of $e_{j}$ from the higher base of $e^{*}\left(e_{j}, \lambda\right)-e_{j}$ increases costs by strictly more ${ }^{29}$, so we have a contradiction.

Case (2): suppose that $e^{*}\left(e_{j}, \lambda\right)-e_{j}=e^{*}(0, \lambda)$. This implies that:

$$
v q\left(e^{*}\left(e_{j}, \lambda\right)-e_{j}\right)-v \lambda \Omega_{i}^{\prime}\left(e^{*}\left(e_{j}, \lambda\right)-e_{j}\right)=v q\left(e^{*}(0, \lambda)\right)-v \lambda \Omega_{i}^{\prime}\left(e^{*}(0, \lambda)\right),
$$

so, given $e^{*}(0, \lambda)>0$ from Lemma 2 and $C^{\prime \prime}\left(e_{i}\right)>0$, from the FOCs we immediately get a contradiction.

$29 \overline{\frac{d(C(x+y)-C(x))}{d x}=C^{\prime}(x+y)-C^{\prime}(x)>0 \text { for } x} \geq 0$ and $y>0$. 
Now let $e^{* *}(\lambda) \equiv e^{*}\left(e^{*}(0, \lambda), \lambda\right)$. Let $\bar{\lambda}$ be the $\lambda$ such that $\lambda\left[1-2 Q\left(-e^{*}(0, \lambda)\right)\right]=1$. As $e^{*}(0, \lambda)>0$ is increasing in $\lambda$ from Lemma $2, Q^{\prime}(x)>0$ and $Q(x) \in\left(0, \frac{1}{2}\right)$ for $x<0$, we see that $1-2 Q\left(-e^{*}(0, \lambda)\right) \in(0,1)$ and is increasing in $\lambda$, so such a $\bar{\lambda}$ exists and is unique. For $\lambda>\bar{\lambda}$, let $\widehat{e}(\lambda)$ be the $e_{i}$ such that $\lambda\left[1-2 Q\left(e_{i}-e^{*}(0, \lambda)\right)\right]=1$. Since $Q(x) \rightarrow \frac{1}{2}$ as $x \rightarrow 0$, $\widehat{e}(\lambda) \in\left(0, e^{*}(0, \lambda)\right)$ and is unique. Further, $\widehat{e}(\lambda)$ is strictly increasing in $\lambda$ and unbounded above: as $\lambda$ goes up, so does $e^{*}(0, \lambda)$, so $\widehat{e}(\lambda)$ needs to rise by even more. Thus, as $e^{*}(0, \lambda)$ is unbounded, so is $\widehat{e}(\lambda)$.

(i) We will show that for $\lambda$ large enough, neither $(\mathrm{a}) e^{* *}(\lambda) \geq \widehat{e}(\lambda)$ nor $(\mathrm{b}) e^{* *}(\lambda) \in(0, \widehat{e}(\lambda))$ is possible, so given a global optimum always exists (see footnote 15), $e^{* *}(\lambda)=0$.

Case (a): Suppose $e^{* *}(\lambda) \geq \widehat{e}(\lambda)$. Letting $\Delta E U_{i}\left(e_{i}, e_{j}, \lambda\right) \equiv E U_{i}\left(e_{i}, e_{j}, \lambda\right)-E U_{i}\left(0, e_{j}, \lambda\right)$ :

$$
\begin{aligned}
\Delta E U_{i}\left(e^{* *}(\lambda), e^{*}(0, \lambda), \lambda\right)= & v\left[Q\left(e^{* *}(\lambda)-e^{*}(0, \lambda)\right)-Q\left(-e^{*}(0, \lambda)\right)\right] \\
& -v \lambda\left[\Omega_{i}\left(e^{* *}(\lambda)-e^{*}(0, \lambda)\right)-\Omega_{i}\left(-e^{*}(0, \lambda)\right)\right] \\
& -\left[C\left(e^{* *}(\lambda)\right)-C(0)\right] .
\end{aligned}
$$

The first term of (16) is bounded above by $v$ since $Q$ is a c.d.f. The second term is strictly negative, as $\left|e^{* *}(\lambda)-e^{*}(0, \lambda)\right|<\left|-e^{*}(0, \lambda)\right|$ and $\Omega_{i}$ is strictly quasi-concave and symmetric about zero from Lemma 1 . Where $e^{* *}(\lambda)<e^{*}(0, \lambda)$, the inequality is automatic, while where $e^{* *}(\lambda) \geq e^{*}(0, \lambda), e^{* *}(\lambda)-e^{*}(0, \lambda)<e^{*}(0, \lambda)$ from $(15)$. Thus $\Delta E U_{i}\left(e^{* *}(\lambda), e^{*}(0, \lambda), \lambda\right)<$ $v-C\left(e^{* *}(\lambda)\right) \leq v-C(\widehat{e}(\lambda))$. As $\widehat{e}(\lambda)$ is unbounded above as $\lambda$ rises and $C^{\prime \prime}\left(e_{i}\right)>0$, for sufficiently large $\lambda \Delta E U_{i}\left(e^{* *}(\lambda), e^{*}(0, \lambda), \lambda\right)<0$, a contradiction as $i$ would then prefer to set zero effort.

Case (b): Suppose that $e^{* *}(\lambda) \in[0, \widehat{e}(\lambda))$. Now, at $e_{i}=e^{* *}(\lambda)$ :

$$
\begin{aligned}
\frac{\partial E U_{i}\left(e_{i}, e^{*}(0, \lambda), \lambda\right)}{\partial e_{i}} & =v q\left(e^{* *}(\lambda)-e^{*}(0, \lambda)\right)-v \lambda \Omega_{i}^{\prime}\left(e^{* *}(\lambda)-e^{*}(0, \lambda)\right)-C^{\prime}\left(e^{* *}(\lambda)\right) \\
& =v q\left(e^{* *}(\lambda)-e^{*}(0, \lambda)\right)\left\{1-\lambda\left[1-2 Q\left(e^{* *}(\lambda)-e^{*}(0, \lambda)\right)\right]\right\}-C^{\prime}\left(e^{* *}(\lambda)\right) .
\end{aligned}
$$

Since $e^{* *}(\lambda)<\widehat{e}(\lambda), \lambda\left[1-2 Q\left(e^{* *}(\lambda)-e^{*}(0, \lambda)\right)\right]>1$, so $\frac{\partial E U_{i}\left(e_{i}, e^{*}(0, \lambda), \lambda\right)}{\partial e_{i}}<0$ at $e_{i}=e^{* *}(\lambda)$, and hence we have a contradiction unless $e^{* *}(\lambda)=0$.

(ii) We start by showing that for small enough $\widetilde{e}_{j}>0, e_{i}=0$ remains the unique global optimum in response to $e_{j}=e^{*}(0, \lambda) \pm \widetilde{e}_{j}$, so where $i$ is the slacker, her reaction function is locally vertical in $\left(e_{i}, e_{j}\right)$ space. First, consider $e_{i} \geq \widehat{e}(\lambda)$. For large enough $\lambda, \Delta E U_{i}\left(e_{i}, e^{*}(0, \lambda), \lambda\right)<0$ as $e^{* *}(\lambda)=0$ is the unique global optimum from above. Furthermore, $\max _{e_{i} \geq \widehat{e}(\lambda)} \Delta E U_{i}\left(e_{i}, e^{*}(0, \lambda), \lambda\right)$ exists as $E U_{i}\left(e_{i}, e^{*}(0, \lambda), \lambda\right)$ is unbounded below as $e_{i}$ goes up. Thus we can find a small enough $\widetilde{e}_{j}$ such that $\Delta E U_{i}\left(e_{i}, e^{*}(0, \lambda) \pm \widetilde{e}_{j}, \lambda\right)<0 \forall e_{i} \geq \widehat{e}(\lambda)$. Second, consider $e_{i}<\widehat{e}(\lambda)$. From 
above, $\frac{\partial E U_{i}\left(e_{i}, e^{*}(0, \lambda), \lambda\right)}{\partial e_{i}}<-C^{\prime}\left(e_{i}\right) \leq 0$. Now $\frac{\partial E U_{i}\left(e_{i}, e^{*}(0, \lambda), \lambda\right)}{\partial e_{i}} \leq \max _{e_{i}<\widehat{e}(\lambda)} \frac{\partial E U_{i}\left(e_{i}, e^{*}(0, \lambda), \lambda\right)}{\partial e_{i}}<0$, so we can find a small enough $\widetilde{e}_{j}$ that $\frac{\partial E U_{i}\left(e_{i}, e^{*}(0, \lambda) \pm \widetilde{e}_{j}, \lambda\right)}{\partial e_{i}}<0 \forall e_{i}<\widehat{e}(\lambda)$. (If the slope is greater at $\widehat{e}(\lambda)$ than any $e_{i}<\widehat{e}(\lambda)$, no maximum will exist on our range, but then $\frac{\partial E U_{i}\left(e_{i}, e^{*}(0, \lambda), \lambda\right)}{\partial e_{i}}<$ $-C^{\prime}(\widehat{e}(\lambda))<0$.) Asymptotic stability follows immediately given the reaction function of the slacker $i$ is locally vertical in $\left(e_{i}, e_{j}\right)$ space and the high effort agent $j^{\prime}$ s reaction function has a locally well-defined finite slope. This last follows from assuming $e^{*}\left(e_{j}, \lambda\right)$ changes smoothly in $e_{j}$ at $e_{j}=0$.

Proof of Proposition 5. Using (1), (2) and (3), and remembering that $\widetilde{P}_{i}\left(e_{i}, e_{j}\right)=$ $Q\left(e_{i}-e_{j}+\theta k\right)$ and $\widetilde{P}_{j}\left(e_{i}, e_{j}\right)=1-\widetilde{P}_{i}\left(e_{i}, e_{j}\right)$, at a symmetric equilibrium $e_{i}=e_{j}=e^{*}$ :

$$
\begin{aligned}
\frac{\partial E U_{i}}{\partial e_{i}} & =v q(k)-v \lambda\left[q(k)\left(1-\widetilde{P}_{i}\right)-q(\theta k) P_{i}\right]-v l[q(\theta k)-q(k)]-C^{\prime}\left(e^{*}\right) \\
\frac{\partial E U_{j}}{\partial e_{j}} & =v q(k)-v \lambda\left[q(k)\left(1-\widetilde{P}_{j}\right)-q(\theta k) P_{j}\right]-v l[q(\theta k)-q(k)]-C^{\prime}\left(e^{*}\right) .
\end{aligned}
$$

When $e_{i}=e_{j}, P_{i}>P_{j}$ due to $i^{\prime} s$ advantage, $\widetilde{P}_{i}>\widetilde{P}_{j}$ for $\theta>0$ and $\widetilde{P}_{i}=\widetilde{P}_{j}$ for $\theta=0$, so

$$
\frac{\partial E U_{i}}{\partial e_{i}}-\frac{\partial E U_{j}}{\partial e_{j}}=v \lambda\left[q(k)\left(\widetilde{P}_{i}-\widetilde{P}_{j}\right)+q(\theta k)\left(P_{i}-P_{j}\right)\right]>0
$$

and hence we cannot have an interior symmetric equilibrium as $\frac{\partial E U_{i}}{\partial e_{i}}$ and $\frac{\partial E U_{j}}{\partial e_{j}}$ can't both equal zero.

When $e_{i}=e_{j}, P_{i} \geq \widetilde{P}_{i}$ and $\widetilde{P}_{i} \geq \frac{1}{2}$. Thus $P_{i} \geq 1-\widetilde{P}_{i}$, so when $\theta=1$ a corner symmetric equilibrium is not possible as $\frac{\partial E U_{i}}{\partial e_{i}}=v q(k)\left[1+\lambda\left(P_{i}-\left(1-\widetilde{P}_{i}\right)\right)\right]-C^{\prime}(0)>0$ given our assumption that $v q(k)>C^{\prime}(0)$. By continuity, this must remain true for $\theta$ sufficiently close to one.

Proof of Proposition 6. Using (1), (2), (3), (8), (9) and (10), and remembering that $\widetilde{P}_{j}=1-\widetilde{P}_{i}$ :

$$
\begin{aligned}
\frac{\partial E U_{i}}{\partial e_{i}} & =\frac{v}{2 \gamma}+v \lambda\left[-\frac{1}{2 \gamma}+\frac{2\left(e_{i}-e_{j}\right)+(1+\theta) k+2 \gamma}{4 \gamma^{2}}\right]-c e_{i} ; \\
\frac{\partial E U_{j}}{\partial e_{j}} & =\frac{v}{2 \gamma}+v \lambda\left[-\frac{1}{2 \gamma}+\frac{2\left(e_{j}-e_{i}\right)-(1+\theta) k+2 \gamma}{4 \gamma^{2}}\right]-c e_{j} ; \\
\frac{\partial^{2} E U_{i}}{\partial\left(e_{i}\right)^{2}} & =\frac{\partial^{2} E U_{j}}{\partial\left(e_{j}\right)^{2}}=\frac{v \lambda}{2 \gamma^{2}}-c .
\end{aligned}
$$

Assumption (d) implies that $2 \gamma^{2} c>v \lambda$, so the objective functions are globally concave. To verify this, note that $2 \gamma^{2} c>\frac{4 \gamma^{2} c(\gamma-k) \lambda}{2 \gamma+\lambda(2 \gamma-(1-\theta) k)} \Leftrightarrow 2 \gamma+\lambda k(1+\theta)>0$. Thus we get the following 
linear reaction functions:

$$
\begin{aligned}
& e_{i}^{*}\left(e_{j}\right)=\left\{\begin{array}{ll}
\frac{2 v \gamma+v \lambda(1+\theta) k-2 v \lambda e_{j}}{2\left(2 \gamma^{2} c-v \lambda\right)} & \text { if } e_{j}<\frac{2 \gamma+\lambda(1+\theta) k}{2 \lambda} \\
0 & \text { if } e_{j} \geq \frac{2 \gamma+\lambda(1+\theta) k}{2 \lambda}
\end{array} ;\right. \\
& e_{j}^{*}\left(e_{i}\right)= \begin{cases}\frac{2 v \gamma-v \lambda(1+\theta) k-2 v \lambda e_{i}}{2\left(2 \gamma^{2} c-v \lambda\right)} & \text { if } e_{i}<\frac{2 \gamma-\lambda(1+\theta) k}{2 \lambda} \\
0 & \text { if } e_{i} \geq \frac{2 \gamma-\lambda(1+\theta) k}{2 \lambda}\end{cases}
\end{aligned}
$$

Notice that $e_{i}^{*}(0) \leq \gamma-k$ iff (d) holds, so $e_{i}^{*}\left(e_{j}\right) \leq \gamma-k$ and $e_{j}^{*}\left(e_{i}\right) \leq \gamma-k$ everywhere given $e_{i}^{*}$ is falling in $e_{j}$ and $e_{j}^{*}(x) \leq e_{i}^{*}(x)$ for all $x$, and hence (b) does not bind on the effort choices.

Now, $e_{i}^{*}(0)=\frac{2 v \gamma+v \lambda(1+\theta) k}{2\left(2 \gamma^{2} c-v \lambda\right)} \lesseqgtr \frac{2 \gamma-\lambda(1+\theta) k}{2 \lambda} \Leftrightarrow 2 v \lambda \lesseqgtr 2 \gamma^{2} c-\lambda \gamma c(1+\theta) k \Leftrightarrow \lambda \lesseqgtr \frac{2 \gamma^{2} c}{2 v+\gamma c(1+\theta) k}$. Also, $e_{j}^{*}(0)=\frac{2 v \gamma-v \lambda(1+\theta) k}{2\left(2 \gamma^{2} c-v \lambda\right)}<\frac{2 \gamma+\lambda(1+\theta) k}{2 \lambda} \Leftrightarrow 2 v \lambda<2 \gamma^{2} c+\lambda \gamma c(1+\theta) k$. If $2 v \leq \gamma c(1+\theta) k$, the inequality must hold. If $2 v>\gamma c(1+\theta) k$, it holds iff $\lambda<\frac{2 \gamma^{2} c}{2 v-\gamma c(1+\theta) k}$.

When $\lambda<\frac{2 \gamma^{2} c}{2 v+\gamma c(1+\theta) k}$, finding the intersection of the reaction functions gives:

$$
\left(e_{i}^{*}, e_{j}^{*}\right)=\left(\frac{2 v\left(\gamma^{2} c-v \lambda\right)+v \lambda \gamma c(1+\theta) k}{4 \gamma c\left(\gamma^{2} c-v \lambda\right)}, \frac{2 v\left(\gamma^{2} c-v \lambda\right)-v \lambda \gamma c(1+\theta) k}{4 \gamma c\left(\gamma^{2} c-v \lambda\right)}\right) .
$$

$\lambda<\frac{2 \gamma^{2} c}{2 v+\gamma c(1+\theta) k}$ implies that the numerator of $e_{j}^{*}$ is strictly positive. Also, $\lambda<\frac{2 \gamma^{2} c}{2 v+\gamma c(1+\theta) k} \Rightarrow$ $\lambda<\frac{2 \gamma^{2} c}{2 v}$ so the denominator is also strictly positive. Thus, $e_{j}^{*}>0$, which implies $e_{i}^{*}>0$. When $k=0$, the equilibrium is symmetric. When $k>0$, the effort difference, $e_{i}^{*}-e_{j}^{*}=\frac{v \lambda(1+\theta) k}{2\left(\gamma^{2} c-v \lambda\right)}>0$, is increasing in $k, \lambda$, and $\theta$.

When $\lambda \geq \frac{2 \gamma^{2} c}{2 v+\gamma c(1+\theta) k}$, and $\lambda<\frac{2 \gamma^{2} c}{2 v-\gamma c(1+\theta) k}$ if $2 v>\gamma c(1+\theta) k$,

$$
\left(e_{i}^{*}, e_{j}^{*}\right)=\left(\frac{2 v \gamma+v \lambda(1+\theta) k}{2\left(2 \gamma^{2} c-v \lambda\right)}, 0\right) .
$$

This case can arise only if $k>0 . e_{i}^{*}>0$ given $2 \gamma^{2} c>v \lambda$ by global concavity. Again, the effort difference is increasing in $k, \lambda$, and $\theta$. We need to check that the $\lambda$ range in (ii) is consistent with (d), so case (ii) is possible: (d) holds iff $\lambda \leq \frac{2 \gamma(2 \gamma c(\gamma-k)-v)}{v(2 \gamma-(1-\theta) k)}$, and $\frac{2 \gamma(2 \gamma c(\gamma-k)-v)}{v(2 \gamma-(1-\theta) k)}>\frac{2 \gamma^{2} c}{2 v+\gamma c(1+\theta) k} \Leftrightarrow$ $[v+\gamma c(1+\theta) k][v-\gamma c(\gamma-k)]<0 \Leftrightarrow v<\gamma c(\gamma-k)$.

In both cases, the equilibrium is asymptotically stable as $e_{i}^{*}\left(e_{j}\right)$ cuts $e_{j}^{*}\left(e_{i}\right)$ from above in $\left(e_{i}, e_{j}\right)$ space.

Proof of Claim 1. Using (17) and allowing for the extra term in $C\left(e_{i}\right)$ :

$$
\begin{aligned}
\frac{\partial E U_{i}}{\partial e_{i}} & =\frac{1}{2}+\frac{1}{2}\left(\frac{e_{i}-e_{j}}{2}\right)-c e_{i}-\frac{2}{5} ; \\
\frac{\partial^{2} E U_{i}}{\partial\left(e_{i}\right)^{2}} & =\frac{1}{4}-c .
\end{aligned}
$$


Suppose first that $c=\frac{2}{5}$. Then the objective functions are strictly concave and the reaction functions are linear. The unique symmetric equilibrium is given by $e_{i}^{*}=e_{j}^{*}=\frac{1 / 2-2 / 5}{2 / 5}=\frac{1}{4}$. However, this equilibrium is unstable as, referring back to Proposition $2, \lambda \in\left[\frac{2 / 5}{4(1 / 2)^{2}}, \frac{2 / 5}{2(1 / 2)^{2}}\right]=$ $\left[\frac{2}{5}, \frac{4}{5}\right]$. There are also two asymmetric equilibria with $\left(e_{i}^{*}, e_{j}^{*}\right) \in\left\{\left(0, \frac{2}{3}\right),\left(\frac{2}{3}, 0\right)\right\}$. To see this, note that if $e_{j}=0, e_{i}^{*}=\frac{1 / 2-2 / 5}{2 / 5-1 / 4}=\frac{2}{3}$, while if $e_{j}=\frac{2}{3}, \frac{\partial E U_{i}\left(0, \frac{2}{3}\right)}{\partial e_{i}}=\frac{1}{2}+\frac{1}{2}\left(\frac{-2 / 3}{2}\right)-\frac{2}{5}=-\frac{1}{15}<0$, so $e_{i}^{*}=0$ given the objective functions are strictly concave. The linearity of the reaction functions ensures there are no other asymmetric equilibria and that these equilibria are asymptotically stable.

Suppose second that $c=\frac{1}{5}$. Then the objective functions are strictly convex, so $e_{i}^{*}\left(e_{j}\right) \in$ $\{0,1\} \forall e_{j}$, and hence the only candidates for equilibrium are given by $\left(e_{i}^{*}, e_{j}^{*}\right) \in\{(0,0),(1,1)$, $(0,1),(1,0)\} \cdot(0,0)$ is not an equilibrium as $\frac{\partial E U_{i}(0,0)}{\partial e_{i}}=\frac{1}{2}-\frac{2}{5}>0 .(1,1)$ is not an equilibrium as $\frac{\partial E U_{i}(1,1)}{\partial e_{i}}=\frac{1}{2}-\frac{1}{5}-\frac{2}{5}<0$. The only equilibria are given by $\left(e_{i}^{*}, e_{j}^{*}\right) \in\{(0,1),(1,0)\}$. If $e_{i}=0$ and $e_{j}=1$, then $i$ will not want to deviate to $e_{i}=1$ as (using (4)) $E U_{i}(0,1)=$ $0>E U_{i}(1,1)=\frac{1}{2}-\left(\frac{1}{2}\right)^{3}-\frac{1 / 5}{2}-\frac{2}{5}=-\frac{1}{8}$, and $j$ will not want to deviate to $e_{j}=0$ as $E U_{j}(1,0)=1-\frac{1 / 5}{2}-\frac{2}{5}=\frac{1}{2}>E U_{j}(0,0)=\frac{1}{2}-\left(\frac{1}{2}\right)^{3}=\frac{3}{8}$. The strict convexity of the objectives ensures we need not consider other deviations, and together with the continuity of the objectives ensures asymptotic stability of the equilibria. There is nothing special about the agents have winning probabilities of exactly one and zero. If we pushed the effort bound a little below one, we would still get the asymmetric equilibria, but the agents would have interior winning probabilities.

Proof of Proposition 7. Under LRPP, using (11) and (12):

$$
\begin{aligned}
w_{i}-r_{i} & =\alpha\left[\left(\psi_{i}-e_{i}\right)-\left(\psi_{j}-e_{j}\right)\right]=\alpha\left[\epsilon_{i}-\epsilon_{j}\right]=-\alpha \eta \\
E U_{i} & =\alpha\left(e_{i}-e_{j}\right)+F_{L R P P}+\int_{-\infty}^{0} g[-\alpha x] q(x) d x+\int_{0}^{\infty} l[-\alpha x] q(x) d x-C\left(e_{i}\right) .
\end{aligned}
$$

As $q(\eta)$ is symmetric about zero, $\int_{0}^{\infty} x q(x) d x=-\int_{-\infty}^{0} x q(x) d x$, and we get:

$$
E U_{i}=\alpha\left(e_{i}-e_{j}\right)-\lambda \alpha \int_{0}^{\infty} x q(x) d x-C\left(e_{i}\right)+F_{L R P P}
$$

$E U_{i}$ is strictly concave, and the first order condition is $\alpha=C^{\prime}\left(e_{i}^{*}\right)$, so each worker setting $e_{i}^{*}=C^{\prime-1}(\alpha)$ is the unique Nash equilibrium. (Of course, if $\alpha \leq C^{\prime}(0)$ we have a corner solution at $e_{i}^{*}=0$.) Note that this is independent of $\lambda$, as given $\alpha$ the expected desert loss is 
the same for any $e_{i}-e_{j}$. To induce total effort of $\widehat{e}$, the employer sets $\alpha=C^{\prime}\left(\frac{\widehat{e}}{2}\right)$, so

$$
E U_{i}=-\lambda C^{\prime}\left(\frac{\widehat{e}}{2}\right) \int_{0}^{\infty} x q(x) d x-C\left(\frac{\widehat{e}}{2}\right)+F_{L R P P}
$$

To satisfy the participation constraint, he must set:

$$
F_{L R P P}(\widehat{e})=\lambda C^{\prime}\left(\frac{\widehat{e}}{2}\right) \int_{0}^{\infty} x q(x) d x+C\left(\frac{\widehat{e}}{2}\right)+\bar{U}
$$

The total wage costs $W_{L R P P}(\widehat{e})$ are simply $2 F_{L R P P}(\widehat{e})$.

Under the tournament, the monetary payoff $y_{i}$ and the reference point $r_{i}$ are both increased by $F_{T}$, so the desert deficit term in $E U_{i}$ is unaffected. Thus, as under the LRPP scheme, conditional on the participation constraint being satisfied behavior is unaltered by the fixed payments. From Propositions 1 and 2, and given our assumption that a symmetric equilibrium exists, to induce total effort $\widehat{e}$ the employer sets $v=\frac{C^{\prime}\left(\frac{\widehat{e}}{2}\right)}{q(0)}$, so using (4):

$$
E U_{i}=\frac{v}{2}-\frac{v \lambda}{4}-C\left(\frac{\widehat{e}}{2}\right)+F_{T}=\frac{C^{\prime}\left(\frac{\widehat{e}}{2}\right)}{2 q(0)}-\frac{\lambda C^{\prime}\left(\frac{\widehat{e}}{2}\right)}{4 q(0)}-C\left(\frac{\widehat{e}}{2}\right)+F_{T} .
$$

To satisfy the participation constraint, he must set:

$$
F_{T}(\widehat{e})=-\frac{C^{\prime}\left(\frac{\widehat{e}}{2}\right)}{2 q(0)}+\frac{\lambda C^{\prime}\left(\frac{\widehat{e}}{2}\right)}{4 q(0)}+C\left(\frac{\widehat{e}}{2}\right)+\bar{U},
$$

and wage costs $W_{T}(\widehat{e})$ are $v+2 F_{T}(\widehat{e})$ so

$$
W_{T}(\widehat{e})=\frac{C^{\prime}\left(\frac{\widehat{e}}{2}\right)}{q(0)}+2\left[-\frac{C^{\prime}\left(\frac{\widehat{e}}{2}\right)}{2 q(0)}+\frac{\lambda C^{\prime}\left(\frac{\widehat{e}}{2}\right)}{4 q(0)}+C\left(\frac{\widehat{e}}{2}\right)+\bar{U}\right]=\frac{\lambda C^{\prime}\left(\frac{\widehat{e}}{2}\right)}{2 q(0)}+2 C\left(\frac{\widehat{e}}{2}\right)+2 \bar{U} .
$$

Using (18) and (19):

$$
W_{T}(\widehat{e}) \lesseqgtr W_{L R P P}(\widehat{e}) \Leftrightarrow 2 \lambda C^{\prime}\left(\frac{\widehat{e}}{2}\right) \int_{0}^{\infty} x q(x) d x \gtreqless \frac{\lambda C^{\prime}\left(\frac{\widehat{e}}{2}\right)}{2 q(0)} .
$$

In the absence of desert $(l=g=\lambda=0)$, LRPP and the tournament have the same cost. With $\lambda>0$, the tournament is cheaper if and only if $4 q(0) \int_{0}^{\infty} x q(x) d x \geq 1$. Because $q(\eta)$ is symmetric about zero,

$$
E[|\eta|]=\int_{0}^{\infty} x q(x) d x+\int_{-\infty}^{0}|x| q(x) d x=\int_{0}^{\infty} 2 x q(x) d x
$$

giving the proposition. 


\section{References}

Adams, J.S. (1965). "Inequity in Social Exchange". In "Advances in Experimental Social Psychology, Vol. II", Berkowitz, L. (Ed.), Academic Press, 267-299.

Barberis, N,. Huang, M. and Thaler, R.H. (2006). "Individual Preferences, Monetary Gambles, and Stock Market Participation: A Case for Narrow Framing". American Economic Review, 96, 10691090.

Bartling, B. and von Siemens, F. (2004). "Efficiency in Team Production with Inequity Averse Agents". Mimeo.

Battigalli, P. and Dufwenberg, M. (2007). "Guilt in Games". American Economic Review: Papers and Proceedings, 97(2), 170-176.

Battigalli, P. and Dufwenberg, M. (2009). "Dynamic Psychological Games". Journal of Economic Theory, 144(1), 1-35.

Bell, D.E. (1985). "Disappointment in Decision Making under Uncertainty". Operations Research, 33, $1-27$.

Bolton, G.E. and Ockenfels, A. (2000). "ERC: A Theory of Equity, Reciprocity and Competition". American Economic Review, 90(1), 166-193.

Bull, C., Schotter, A. and Weigelt, K. (1987). "Tournaments and Piece Rates: An Experimental Study". Journal of Political Economy, 95(1), 1-33.

Burrows, P. and Loomes, G. (1994). "The Impact of Fairness on Bargaining Behaviour". Empirical Economics, 19, 201-221.

Cappelen, A.W., Hole, A.D., Sørensen, E.O. and Tungodden, B. (2007). "The Pluralism of Fairness Ideals: An Experimental Approach". American Economic Review, 97(3), 818-827.

Chowdhury, S.M. and Sheremeta, R.M. (2009). "A Generalized Tullock Contest and the Existence of Multiple Equilibria". Mimeo.

Conyon, M.J., Peck, S.I. and Sadler, G.V. (2001). "Corporate Tournaments and Executive Compensation: Evidence from the U.K.". Strategic Management Journal, 22(8), 805-815.

Cornes, R. and Hartley, R. (2003). "Loss Aversion and the Tullock Paradox". Keele Economics Research Papers 2003/06.

Demougin, D. and Fluet, C. (2003). "Inequity Aversion in Tournaments". CIRPEE Working Paper No. 03-22.

DeVaro, J. (2006). "Internal Promotion Competitions in Firms". RAND Journal of Economics, 37(3), 521-542.

van Dijk, F., Sonnemans, J. and van Winden, F. (2001). "Incentive Systems in a Real Effort Experiment". European Economic Review, 45, 187-214.

Dufwenberg, M. and Kirchsteiger, G. (2004). "A Theory of Sequential Reciprocity". Games and Economic Behavior, 47(2), 268-298.

Ederer, F. and Fehr, E. (2006). "Communication and Information in Dynamic Tournaments: Theory and Evidence". Mimeo.

Englmaier, F. (2005). "A Survey on Moral Hazard, Contracts, and Social Preferences". In "Psychology, Rationality and Economic Behaviour: Challenging Standard Assumptions", Agarwal, B. and Vercelli, A. (Eds.), Palgrave Macmillan, 125-140. 
Falk, A. and Fischbacher, U. (2006). "A Theory of Reciprocity". Games and Economic Behavior, 54(2), 293-315.

Fehr, E. and Schmidt, K. (1999). "A Theory of Fairness, Competition and Cooperation". Quarterly Journal of Economics, 114, 817-868.

Frank, R.H. (2005). "Positional Externalities Cause Large and Preventable Welfare Losses". American Economic Review: Papers and Proceedings, 95(2), 137-141.

Frohlich, N., Oppenheimer, J. and Kurki, A. (2004). "Modeling Other-Regarding Preferences and an Experimental Test". Public Choice, 119, 91-117.

Fudenberg, D. and Tirole, J. (1991). "Game Theory". MIT Press.

Geanakoplos, J., Pearce D. and Stacchetti, E. (1989). "Psychological Games and Sequential Rationality". Games and Economic Behavior, 1(1), 60-79.

Gill, D. and Prowse, V. (2009). "A Structural Analysis of Disappointment Aversion in a Real Effort Competition". Dept. of Economics Discussion Paper 448, University of Oxford.

Gill, D. and Stone, R. (2006). "Fairness and Desert in Tournaments". Dept. of Economics Discussion Paper 279, University of Oxford.

Gneezy, U., List, J.A. and Wu, G. (2006). "The Uncertainty Effect: When a Risky Prospect is Valued Less Than its Worst Possible Outcome". Quarterly Journal of Economics, 121(4), 1283-1309.

Green, J.R. and Stokey, N.L. (1983). "A Comparison of Tournaments and Contracts". Journal of Political Economy, 91(3), 349-364.

Grund, C. and Sliwka, D. (2005). "Envy and Compassion in Tournaments". Journal of Economics and Management Strategy, 14(1), 187-207.

Güth, W. (1988). "On the Behavioral Approach to Distributive Justice - A Theoretical and Experimental Investigation". In "Applied Behavioral Economics, Vol. II.", Maital, S. (Ed.), Wheatsheaf, 703-717.

Harbring, C. and Irlenbusch, B. (2003). "An Experimental Study on Tournament Design". Labour Economics, 10, 443-464.

Hopkins, E. and Kornienko, T. (2004). "Running to Keep in the Same Place: Consumer Choice as a Game of Status". American Economic Review, 94(4), 1085-1107.

Itoh, H. (2004). "Moral Hazard and Other-Regarding Preferences". Japanese Economic Review, 55, $18-45$.

Kahneman, D., Knetsch, J.L. and Thaler, R. (1986). "Fairness as a Constraint on Profit Seeking: Entitlements in Markets". American Economic Review, 76(4), 728-741.

Kahneman, D. and A. Tversky (1979). "Prospect Theory: An Analysis of Decision under Risk." Econometrica, 47, 263-291.

Konow, J. (1996). "A Positive Theory of Economic Fairness". Journal of Economic Behavior and Organization, 31, 13-35.

Konow, J. (2000). "Fair Shares: Accountability and Cognitive Dissonance in Allocation Decisions". American Economic Review, 90(4), 1072-1091.

Konow, J. (2003). "Which is the Fairest One of All? A Positive Analysis of Justice Theories". Journal of Economic Literature, 41, 1188-1239. 
Köszegi, B. and Rabin, M. (2006). "A Model of Reference-Dependent Preferences". Quarterly Journal of Economics, 121(4), 1133-1165.

Köszegi, B. and Rabin, M. (2007). "Reference-Dependent Risk Attitudes". American Economic Review, 97(4), 1047-1073.

Lazear, E.P. and Rosen, S. (1981). "Rank-Order Tournaments as Optimal Labor Contracts". Journal of Political Economy, 89(5), 841-864.

Loomes, G. and Sugden, R. (1986). "Disappointment and Dynamic Consistency in Choice under Uncertainty". Review of Economic Studies, 53, 271-282.

Malcomson, J.M. (1984). "Work Incentives, Hierarchy, and Internal Labor Markets". Journal of Political Economy, 92(3), 486-507.

McLaughlin, K.J. (1988). "Aspects of Tournament Models: A Survey". In "Research in Labor Economics, Vol. 9", Ehrenberg, R. (Ed.), JAI Press, 225-256.

Mellers, B., Schwartz A. and Ritov, I. (1999). "Emotion-Based Choice". Journal of Experimental Psychology: General, 128(3), 332-345.

Nalebuff, B.J. and Stiglitz, J.E. (1983). "Prizes and Incentives: Towards a General Theory of Compensation and Competition". Bell Journal of Economics, 14(1), 21-43.

Prendergast, C. (1999). "The Provision of Incentives in Firms". Journal of Economic Literature, 37(1), $7-63$.

Rabin, M. (1993). "Incorporating Fairness into Game Theory and Economics". American Economic Review, 83(5), 1281-1302.

Rabin, M. (1998). "Psychology and Economics". Journal of Economic Literature, 36(1), 11-46.

Rey Biel, P. (2008) "Inequity Aversion and Team Incentives". Scandinavian Journal of Economics, 110(2), 297-320.

Schotter, A. and Weigelt, K. (1992). "Asymmetric Tournaments, Equal Opportunity Laws, and Affirmative Action: Some Experimental Results". Quarterly Journal of Economics, 107(2), 511-539.

Sheremeta, R.M. (2009) "Experimental Comparison of Multi-Stage and One-Stage Contests". Games and Economic Behavior, forthcoming (doi:10.1016/j.geb.2009.08.001)

Stone, R. (2006). "Self-handicapping, Tournaments and Ego Utility". Mimeo. 